\title{
Optimal Contracts For Providing Load-Side Frequency Regulation Service Using Fleets of Electric Vehicles
}

\author{
Hadi Zarkoob ${ }^{\mathrm{a}, *}$, Srinivasan Keshav ${ }^{\mathrm{b}}$, Catherine Rosenberg ${ }^{\mathrm{b}}$ \\ ${ }^{a}$ Stanford University, 450 Serra Mall Stanford, CA 94305, USA \\ ${ }^{b}$ University of Waterloo, 200 University Ave. West, Waterloo, ON N2L 3G1, Canada
}

\section{Abstract}

We focus on the charging process of a fleet of electric vehicles overnight for providing load-side regulation service. At the heart of this complex problem, the goal is to transfer a certain amount of energy to the fleet by a given deadline; however, when and how fast the energy is sent is flexible. We examine a unidirectional setting in the cases where regulation signals are deterministic (worst case) and stochastic. We study both a single-shot optimization scenario carried at the start of the charging period, and a dynamic optimization scenario, where the optimal control strategy is re-evaluated several times over the duration of the charging interval. We show that most of the gains from dynamic optimization can be obtained by re-evaluating the optimization problem at the midpoint of the charging interval. Moreover, the optimal value of the regulation service in the worst-case deterministic setting nearly matches the stochastic setting with dynamic optimization. We validate our

\footnotetext{
*Corresponding author. Tel: +1 (650) 2233955

Email addresses: hzarkoob@stanford.edu (Hadi Zarkoob), keshav@uwaterloo.ca (Srinivasan Keshav), cath@uwaterloo.ca (Catherine Rosenberg)
} 
results using both simulation and real-world data.

Keywords: Vehicle-to-grid, Load-side frequency regulation service, Ancillary service, Variable-rate charging

\section{Introduction and State of the Art}

\subsection{Introduction}

The frequency regulation service, one of the key ancillary services in the power grid, balances generation and load, taking control action as frequently as once every 30 seconds. Without this balance, alternating current frequency deviates from its standard value (for example, $60 \mathrm{~Hz}$ in North America), which can hurt grid-connected equipment, and, in the worst case, permanently damage generators. Currently, frequency regulation service is provided by a set of generators contracted to respond rapidly to control signals to increase or decrease their power. If the amount of generated power is increased to compensate for the excess grid load, the service is called regulation up. On the other hand, if the amount of generated power is decreased to match the reduced load in the grid, the service is called regulation down.

The balance between generation and load can be equivalently achieved by changing the aggregated load, provided these loads have some level of flexibility in their consumption profile. This approach has several benefits:

- Generators that provide frequency regulation service are typically natural gas or hydroelectric generators. That is because, unlike coal and 


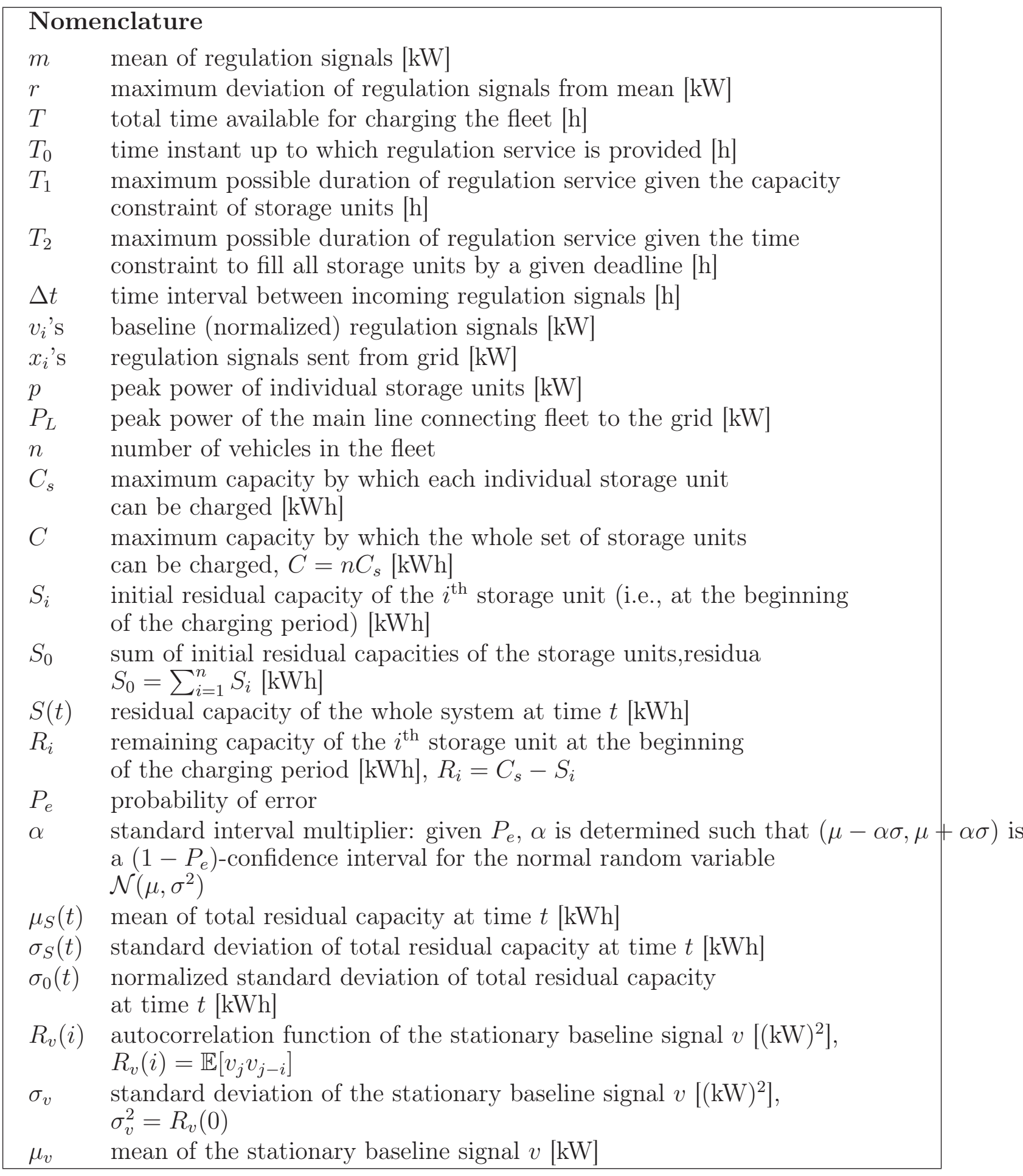




\begin{tabular}{|ll|}
\hline Nomenclature \\
$T_{C}$ & correlation time of the regulation signal $x[\mathrm{~h}]$ \\
$f_{m, r}^{\text {up }}(t)$ & upper bound on the system residual capacity at time $t[\mathrm{kWh}]$ \\
$f_{m, r}^{\text {down }}(t)$ & lower bound on the system residual capacity at time $t[\mathrm{kWh}]$ \\
$P_{C}$ & average power necessary for charging the fleet $[\mathrm{kW}], P_{C}=\frac{C-S_{0}}{T}$ \\
$Q$ & $Q$ is called power ratio and defined as $Q=\frac{P_{C}}{P_{L} / 2}(\mathrm{unitless})$ \\
$\kappa$ & a constant defined as product of $\alpha$ and $\sigma_{v}$ squared $\left[(\mathrm{kW})^{2}\right], \kappa=\left(\alpha \sigma_{v}\right)^{2}$ \\
$T_{u}$ & time interval between update points in the dynamic charging $[\mathrm{h}]$ \\
$d$ & number of update points in the dynamic charging \\
$m^{i}$ & value of mean charge rate decided at time \\
$r_{i}^{i}$ & $t_{i}=i T_{u}[\mathrm{~kW}]$ \\
$T_{0}^{i}$ & value of maximum deviation from mean $m^{i}$ decided \\
$T_{\text {reg }}^{i}$ & at time $t_{i}=i T_{u}[\mathrm{~kW}]$ \\
$m^{\text {opt }}$ & startinal duration of frequency regulation service $t_{i}=i T_{u}[\mathrm{~h}]$ \\
$r^{\text {opt }}$ & realized duration of frequency regulation service \\
$T_{0}^{\text {opt }}$ & optimal value of the mean charge rate $[\mathrm{kW}]$ \\
& optimal value of the maximum deviation from mean $[\mathrm{kW}]$ \\
\end{tabular}


nuclear generators, the output of these generators can be easily adjusted by changing the level of the input gas or water. The problem with gas generators is that they burn fossil fuels, thus causing economical and environmental problems. Hydroelectric generators do not burn fossil fuels. However, a generator that is planned to provide regulation service has to work below its maximum capacity to create room for manoeuvring its output. As a result, if a hydroelectric generator is used for providing regulation, some capacity for generating clean energy will be lost.

- Generators typically achieve maximum efficiency when working at maximum capacity. However, a generator providing balancing service necessarily works, on average, below its maximum capacity, and thus does not achieve its maximum efficiency.

- Generating variable-rate power leads to higher wear and tear of generators. In contrast, some loads may be insensitive or less sensitive to the variations in the input power, and are therefore better candidates for providing regulation service.

- With the advent of new sources of renewable generation and variable load in the future grid, the need for regulation will increase. Being equipped with control units, some loads in the future smart grid can actively participate in the regulation service market.

For a load to be able to participate in the frequency regulation market, three 
conditions must be met: First, it must have some level of flexibility in its consumption profile and therefore be relatively insensitive to variations in input power. Second, the load must be significant with respect to the power fluctuations in the grid, because the utility does not want to deal with minor players. Third, the load should be controllable, so that it can respond to regulation control signals. Examples of such loads include industrial cold storage units, industrial boilers, large-scale pumps and ventilators, and storage units of fleets of electric vehicles. Over the past years, pilot programs have demonstrated successful application of load-side regulation service providers [8]. This has even motivated commercial entities to monetize the aggregation of loads to provide regulation service [9].

In this paper, we focus on the charging process of a fleet of electric vehicles overnight as a representative system for providing load-side regulation service. This system provides a simple setting to study this complex problem. At its heart, the goal is to transfer a certain amount of energy to the fleet by a given deadline; however, when and how fast the energy is sent is flexible [9]. Studying fleets also allows us to analyze a simple multi-component systems (each vehicle in the fleet is one component of the overall system). Studying multi-component loads is important in the context of load-side frequency regulation service because, as mentioned earlier, for a load to take part in the frequency regulation market, it needs to have a consumption rate that is significant with respect to the power fluctuations in the grid. A coalition of multiple loads may be required to achieve this minimum required input 
power.

The integration of electric vehicles with the power grid to achieve a mutual benefit is termed vehicle-to-grid (V2G) [2]. This integration may take two forms: bidirectional and unidirectional. In bidirectional integration, electric vehicles can both receive energy from the grid and send energy to it depending on the consumption profile of other loads in the network. In the unidirectional case, electric vehicles can only receive energy from the grid. In this case, electric vehicles can still be used for providing both regulation up and regulation down services. This is because the input power to the electric vehicles can be decreased or increased depending on the status of other loads in the network. Due to the relatively high loss currently associated with AC-DC and DC-AC power transfers, the bidirectional case is thought to be infeasible in practice. In contrast, in the unidirectional case, no extra infrastructure other than the communication and control system is required [16]. In this work, we therefore focus on unidirectional charging of electric vehicles.

\subsection{Relationship to prior work}

The use of electric vehicles for providing frequency regulation service has received much attention during the recent years, and it has been studied by researchers from different perspectives [14-23]. Authors in [19, 23] use dynamic programming to obtain optimal regulation signal bids. The algorithms proposed in these studies aim to maximize the value of regulation 
service while providing electric vehicles with the desired target residual capacities 1 . One limitation of the above studies is that the variation in system residual capacity due to regulation up and down signals is assumed to be negligible. In other words, it is assumed that the accumulative sum of regulation up and down signals is ideally equal to zero. As we will see in this study, regulation up and down signals are random in nature, and even if they have zero mean, they have a non zero variance. Accounting for this variance is important in obtaining reliable estimates of the system performance. Sortomme and El-Sharkawi [16, 10] study unidirectional frameworks for providing frequency regulation service, and extend their analysis to bidirectional settings in [18]. The formulation presented in these studies allows regulation up and regulation down signals to have unequal expected values. However, these formulations also do not account for the variance of the system residual capacity due to the random nature of regulation up and down signals. It should be noted that as time goes on the uncertainty in the system residual capacity increases, due to the random nature of the regulation signals. That is because regulation signals are added to each other over time and their uncertainties compound. Kamboj et al. [14, 1] study the formation of electric vehicle coalitions and propose heuristic algorithms for determining the average charge rate and regulation service bids. Although algorithms proposed in these studies are reasonable choices, they are not guaranteed

\footnotetext{
${ }^{1}$ The residual capacity of a storage unit is defined as the total energy stored in the unit. It is measured in units of energy, kWh.
} 
to result in the optimal value of regulation service in the system. This is especially the case in unidirectional settings where charging of electric vehicles and provision of regulation service should be done simultaneously, and it is not clear how to select system parameters to effectively achieve both these goals. In this study, we aim to develop a mathematical framework that can be used to obtain rigorous values of the system parameters given the constraints imposed by both utility and owners of electric vehicle. Another limitation of the above studies is that they all compute regulation service bids solely based on constraints of the individual battery units. In practice, however, the main line connecting the aggregator to the utility also has a maximum tolerable power.

In this work, we present a framework to model regulation signals and we study it both in a worst case deterministic setting and a stochastic setting.

\subsection{Contributions}

The contributions of this work are four fold:

1. We introduce a framework to model regulation signals in a system that provides load-side frequency regulation service. This framework captures the performance boost gained from exploiting the opposing effect of regulation up and down signals. It also helps to precisely characterize the uncertainty in the system residual capacity.

2. We use an analytical approach to obtain the optimal values of the regulation parameters. We study the system in the cases where regulation 
signals are deterministic (worst case) and stochastic.

3. We use our approach to study both a single-shot optimization scenario carried at the start of the charging period, and a dynamic optimization scenario, where the optimal control strategy is re-evaluated several times over the duration of the charging interval. We show that most of the gains from dynamic optimization can be obtained by reevaluating the optimization problem at the midpoint of the charging interval. Moreover, the optimal value of the regulation service in the worst-case deterministic setting nearly matches the stochastic setting with dynamic optimization.

4. We obtain an analytical condition under which a multi-component system can be modelled as a single large storage system, thus achieving its maximum performance. We also study how to satisfy this condition in practical systems and how the estimates of the system performance should be modified when the desired condition is not satisfied.

Although the results in this work are presented for the special case of charging a fleet of electric vehicles, they address key aspects of load-side frequency regulation service, and can be applied to broader applications in this context, such as large-scale pumps and industrial boilers.

The organization of the paper is as follows: In Section 2, we describe the characteristics of the fleet of electric vehicles that is studied in this work. In Section 3, we explain mathematical models used to describe the incoming regulation signals as well as the system of electric vehicles. In Section 4, we 
analytically obtain a condition under which the assumption that a collection of storage units in the fleet can be modeled as a single large storage unit is valid. In Section 5, we formulate a single-shot optimization problem under this assumption and study this problem under a worst case deterministic setting and a stochastic setting. In Section 6, we introduce a dynamic optimization scenario in which the parameters of the system may be updated multiple times during the charging process, and study how the performance of the system improves as a result of this dynamic charging. In Section 6.2, we present numerical results obtained from both simulation and real-world data taken from PJM Interconnection broader [13]. We conclude the paper in Section 7.

\section{System}

We study a fleet of electric vehicles with a single owner, who contracts with an electric utility (such as a local distribution company or a third-party aggregator) to provide frequency regulation service. From the owner's perspective, it is critical that the vehicles are fully charged before a certain deadline (typically overnight). However, the owner is insensitive to the actual charging rate, as long as this condition is met. Therefore, we propose a contract where control over vehicle charging is ceded to the utility (or aggregator), which can charge the vehicles at will as long as the vehicles are fully charged before the deadline. In return, the fleet owner obtains a monetary reward to offset operating costs. In this work, we do not study the form or 
value of this monetary compensation. Examples of fleets that can be used for this purpose include fleet of vehicles used for courier services, or a fleet of city transit buses.

The utility uses the storage units of the electric vehicles to maintain the balance between generation and load in the grid. More precisely, the fleet has a charge controller that responds to a regulation signal to control the charging rate of the electric vehicles. The value of this signal reflects stochastic fluctuations in other loads in the grid. This naturally implies that the utility needs to stop obeying the regulation signals at some point and switch to a deterministic phase to ensure that all the storage units are fully charged by the end of the charging period.

The value of the regulation service to a utility depends on two factors: the magnitude $r$ of power variations from the mean value $m$, and the duration $T_{0}$ over which the regulation service is provided. In current regulation service markets, the value of regulation service is measured in MW-h. For example, if a generator agrees to vary its output up to $5 \mathrm{MW}$ above or below its mean output for 1 hour, the value of the resulting regulation service will be 5 MW-h. Motivated by these considerations, in our work, the utility will select for the fleet the two regulation parameters $m$ and $r$ to maximize the objective function which is the product of the magnitude of the maximum variation in the regulation signal, i.e., $r$ and the length of the interval over which regulation service is provided, i.e., $T_{0}$.

Batteries are by far the most expensive part of electric vehicles and their 
charging and discharging need to obey a set of tight constraints to deliver the expected lifetime. Typically, the battery management system in the battery pack will tell the charger how much power it is willing to accept (i.e., a maximum limit) at a given time depending on the current state of charge, temperature, age, etc. As a result, our assumption that any regulation signal can be obeyed is very strong. By making it, we obtain results that should be considered as a bound on the performance that can be achieved by practical systems.

\section{Model}

This section presents a mathematical model for the system described in the previous section.

Let the charging process occur over the interval $[0, T]$ (i.e., $T$ is the deadline). Recall that regulation signals are sent periodically by the grid to the charge controller, typically every 30 seconds. To model this phenomenon, we assume that over this interval, the grid sends $k$ regulation signals denoted $x_{1}, \cdots, x_{k}$. The $j^{\text {th }}$ regulation signal $x_{j}, 1 \leq j \leq k$, is used to control fleet charging over the interval $((j-1) \Delta t, j \Delta t)$. The utility, which has been given control of the fleet during this interval, has to determine the regulation parameters $m$ and $r$ where the regulation signals have mean $m$ and may vary up to a maximum amount of $r$ above or below $m$ as well as the time $T_{0}$ during which the regulation service is enabled.

The regulation signal sequence is bounded from below and above by two 
worst case deterministic sequences. The minimal regulation sequence is a sequence of $k$ signals, each with the value $m-r$. Symmetrically, the maximal regulation sequence is a sequence of $k$ signals, each with the value $m+r$. Unlike prior work, which has only studied the charging problem in the presence of the worst case sequences, we study the optimal fleet charging when we model the regulation signal sequence as $x_{j}=m+r v_{j}, 1 \leq j \leq k$, where $v$ denotes a zero-mean stationary stochastic process with maximum +1 and minimum -1. Here, the discrete-time process $v$ is assumed independent of $m$ and $r$ and captures the intrinsic properties of the regulation signal. When dealing with the stochastic case, we will assume that the cumulative sum of $x_{j}$ 's can be modeled as a Gaussian random variable. This is a reasonable assumption, because as we will see in Section 6.2, regulation signals obey a truncated Gaussian distribution, and the correlation time between them is typically considerably less than the length of the regulation period.

The charge controller charges the electric vehicles by obeying the regulation signal sequence $x_{j}$ until time $T_{0}$. In the remaining time, $T-T_{0}$, the utility switches to a deterministic phase and completes the charging process of all storage units in all vehicles by time $T$. This two-phase charging process is required because the utility needs to ensure that all storage units will be fully charged by the end of the charging period regardless of the (uncertain) residual capacity of the system at time $t=T_{0}$.

We denote by $n$ the number of vehicles in the fleet. We use $S_{i}$ to denote the initial residual capacity of the $i^{\text {th }}$ vehicle, $i=1, \cdots, n$. We denote the 
maximum capacity by which each storage unit can be charged by $C_{s}$. We represent the peak rate at which each individual storage unit can be charged by $p$ and the power capacity of the power line connecting the fleet with the grid by $P_{L}$. Using the above notations, the value of the regulation service in the system can be represented as $r T_{0}$.

It is known that both overcharging and overdischarging of batteries of electric vehicles negatively impact their lifetime. As a result, batteries of electric vehicles are usually charged only up to a fraction of their total capacity, e.g. $90 \%$ of the total capacity. Also, they are discharged only up to a minimum charge level, e.g., $10 \%$ of the total capacity. In the above formulation, $C_{s}$ is the maximum charge level up to which each individual storage unit can be charged. This value is always less than the nominal capacity of the storage units. Since, in this work we study a unidirectional setting for charging electric vehicles, the batteries of the electric vehicles are never discharged. As a result, we do not need to be concerned with the minimum charge level of the storage units. We only need to assume that the initial residual capacities of the storage units are such that they satisfy the minimum setpoint constraint.

\section{Equivalence Assumption}

Before formulating our optimization problem, we derive a condition under which we can model the fleet of electric vehicles as a single large storage unit with peak power $P_{L}$, total capacity $C=n C_{s}$ and initial charge $S_{0}=\sum_{i=1}^{n} S_{i}$. 
Theorem 1 below summarizes the results in this section. This theorem provides a sufficient and necessary condition under which the distributed storage system composed of $n$ identical units of size $C_{s}$, each having a maximum charging rate of $p$ and the single large storage unit are equivalent to each other. Equivalence here means that both systems will behave the same irrespective of the regulation signals being sent. In other words, two systems are called equivalent if any signals that can be obeyed by one of them can be obeyed by the other one as well, and vice versa.

What makes a distributed system different from its corresponding single storage unit is the limitation in the input power of individual storage units. As an example, consider a distributed system that has $n=2$ units with $p=1, C_{s}=1$, and with initial residual capacity both equal to 0.5 . Let $P_{L}=2$. We would like to replace this distributed system by a single central unit of size $C=2$ and initial residual capacity equal to 1 . Whenever the charge controller receives a regulation signal, it has to decide how to schedule the charging of the two units. Imagine that it decides to charge unit 1 with higher priority. Then, at some point, unit 1 will be fully charged and the regulation can only be performed on the second unit that can be charged with an input power $p<P_{L}$. Thus, at this point the distributed system will not be equivalent to single unit any more. It is easy to see that if the charge controller has treated each unit identically, the equivalence would have held. As illustrated by this example, both the initial residual capacity of the storage units and the distribution mechanism determine whether the 
two systems are equivalent or not.

It can be seen that the distributed system and the single large storage unit are equivalent to each other only if the input power can be distributed among the storage units in such a way that the number of none-full storage units times $p$ remains greater than or equal to the incoming power during the whole charging process. Theorem 1 below provides a necessary and sufficient condition for this relation to hold. As we will see in the proof of this theorem, Proportional Charging (PC) turns out to be an ideal scheme for distributing the incoming power among the storage units.

Theorem 1. Consider a distributed system composed of $n$ storage units each with capacity $C_{s}$ and peak power $p$. Assume the $i^{\text {th }}$ storage unit in this system has initial residual capacity $S_{i}$, i.e., the capacity to fill is $R_{i}=C_{s}-S_{i}$, $1 \leq i \leq n$. Also, assume the system is connected to the grid through a power line with peak power $P_{L}$.

The above distributed system is equivalent to a single large storage unit with total capacity $C=n C_{s}$, initial residual capacity $S_{0}=\sum_{i=1}^{n} S_{i}$, and input peak power $P_{L}$, if and only if the following condition holds:

$$
\frac{R_{\max }}{p} \leq \frac{\sum_{i=1}^{n} R_{i}}{P_{L}}
$$

where $R_{\text {max }}=\max _{i}\left\{R_{i}\right\}$.

Proof. Recall that in our system, a regulation signal always corresponds 
to charging (never to discharging). It is not hard to see that any signal that can be obeyed by the distributed system can also be obeyed by the single large storage unit system, because its charging rate matches the peak input power. Thus, to show the above equivalence, we just need to show that condition 1 is a sufficient and necessary condition under which any signal that can be obeyed by the single large storage unit can also be obeyed by the distributed system 2 .

First, we prove that condition 1 is sufficient for this equivalence to hold. Consider a regulation signal $q(t)>0$ over the interval $[0, T]$ that is known to be absorbable by the single large storage unit. This implies that signal $q(t)$ satisfies the following two constraints:

$$
\begin{aligned}
q(t) & \leq P_{L}, \text { for all } 0 \leq t \leq T \\
\int_{0}^{T} q(t) d t & \leq R
\end{aligned}
$$

where $R=\sum_{i=1}^{n} R_{i}$ is the remaining capacity in the large storage unit. We show that signal $q(t)$ can also be sent to the distributed storage system if conditions 1, 2 and 3 hold and the PC scheme is used for distributing the incoming power among the individual units. Under the PC scheme the fraction of input power that is sent to each storage unit is proportional to the

\footnotetext{
${ }^{2}$ We say a signal can be obeyed by a storage system if the peak power of the signal is at most as large as the peak input power of the storage unit and also the energy requested by the signal is at most as large as the remaining capacity of the storage unit.
} 
remaining capacity of that unit. It is not hard to see that under this scheme the fraction of power sent to each storage unit does not change over time.

To show signal $q(t)$ can also be obeyed by the distributed system, we first note that the individual peak power of storage units, $p$, will be never violated during the charging process. That is because the fraction of power sent to the $i^{\text {th }}$ storage unit, denoted by $q_{i}(t)$, satisfies:

$$
q_{i}(t)=\frac{R_{i}}{\sum_{i=1}^{n} R_{i}} q(t) \leq \frac{R_{\max }}{\sum_{i=1}^{n} R_{i}} q(t) \leq \frac{R_{\max }}{\sum_{i=1}^{n} R_{i}} P_{L} \leq p
$$

The first inequality in the above expression comes from the fact that $R_{\max }$ is the largest value among all $R_{i}$ 's. The second inequality comes from inequality 2, and the third inequality comes from condition 1 .

Next, we note that none of the storage units becomes fully charged until perhaps the end of the charging process. To show this we note that the total energy send to the $i^{\text {th }}$ storage unit by time $T$, denoted by $E_{i}$, satisfies:

$$
E_{i}=\int_{0}^{T} q_{i}(t) d t=\int_{0}^{T} \frac{R_{i}}{\sum_{i=1}^{n} R_{i}} q(t) d t=\frac{R_{i}}{R} \int_{0}^{T} q(t) d t \leq \frac{R_{i}}{R} R=R_{i}
$$

The facts that the incoming power $q(t)$ can be distributed among the individual units without their peak power being violated, and none of the storage units become fully charged before the the end of the charging process, imply that signal $q(t)$ can be absorbed by the distributed storage system.

We next show that condition 1 is necessary to ensure that the any sig- 
nal that can be sent to the single big storage unit can also be sent to the distributed system. To show this, we consider the constant signal $q(t)=P_{L}$ over the interval $[0, T]$, where $T=\frac{R}{P_{L}}$, and $R$ is the remaining capacity of the big storage unit. The big storage unit can absorb this signal, and at the end of the charging period it will become fully charged. If the big storage unit and the distributed system are equivalent to each other, then the distributed system must also be able to absorb this signal. One should note that if the distributed system absorbs the above signal, then all individual storage units must get fully charged by the end of the charging period. But the maximum energy that can be sent to each storage unit over the interval $\left[0, \frac{R}{P_{L}}\right]$ is $p\left(\frac{R}{P_{L}}\right)$. Thus, for all storage units we must have $R_{i} \leq p \frac{R}{P_{L}}$. In particular, we must have $R_{\max } \leq p \frac{R}{P_{L}}$, where $R_{\max }$ is the maximum remaining capacity among the whole storage units. This completes the proof.

\section{Optimal Single-Shot Charging}

In this section, we formulate the single-shot optimization problem and derive the optimal parameters for the charging process. We study a singleshot charging scheme, where the values of $m, r$ and $T_{0}$ are determined at the beginning of the charging period and do not change until the end of the process. Subsequently, in Section 6, we study a dynamic setting in which the value of the charging parameters can be updated during the charging process.

In this section, we assume that the fleet of electric vehicles can be mod- 
elled as a single large storage unit with input charge power upper bounded by $P_{L}$ and a total capacity $C=n C_{s}$. The initial residual capacity of this system is denoted by $S_{0}=\sum_{i=1}^{n} S_{i}$. We have called this assumption the equivalence assumption earlier. In Section 4, we have analytically obtained a condition under which this assumption is valid, and see that this condition is likely to be satisfied in all practical systems where the initial residual capacity of the batteries is relatively low.

As previously mentioned, the values of $m, r$ and $T_{0}$ should be computed so that the value of regulation service $r T_{0}$ is maximized. In solving this problem, two key constraints must be taken into account: (i) the system must be able to provide regulation service with the contracted deviation parameter $r$ during the whole interval $\left[0, T_{0}\right]$, (ii) the storage will get fully charged by the end of the charging period $T$. Note that these are two fundamental constraints in the system, and similar constraints are expected to exist in other load-side frequency regulation service providers.

To ensure that regulation service is successfully provided over the interval $\left[0, T_{0}\right]$ (constraint (i) described above), the following constraints must be satisfied: $m-r \geq 0$ and $m+r \leq P_{L}$. The first inequality captures the unidirectional nature of the charging process. The second inequality accounts for the bounded size of the link connecting the fleet to the grid. Another constraint is that the system must never get fully charged during the interval $\left[0, T_{0}\right]$; otherwise, it cannot respond to the incoming regulation signals anymore. Let $S(t)$ denote the residual capacity of the system at time $t$. The 
value of $S(t)$ at time $t=l \Delta t, 1 \leq l \leq k$, can be written as:

$$
S(t)=S_{0}+\Delta t \sum_{j=1}^{l} x_{j}
$$

where the $x_{j}$ 's are the regulation signals that are unknown. The value of the system residual capacity at time $T_{0}$ must be less than or equal to $C$. Mathematically, this constraint can be written as $S\left(T_{0}\right) \leq C$.

To guarantee that all vehicles will be fully charged by the end of the charging process (constraint (ii) described above), we need to ensure that there is enough time to fill the remaining capacity of the vehicle batteries, $C-S\left(T_{0}\right)$, during the second phase of the charging process which has duration $T-T_{0}$, knowing that there is a constraint on the peak power of the main line, $P_{L}$. This constraint can be written as $\frac{C-S\left(T_{0}\right)}{P_{L}} \leq T-T_{0}$.

Using the above constrains, the stochastic optimization problem that the utility should solve to select the regulation parameters $m, r$ and $T_{0}$ is: 


$$
\begin{array}{rc}
\max _{m, r, T_{0}, l_{0}} & r T_{0} \\
\text { s.t. } & m, r, T_{0} \geq 0, T_{0} \leq T \\
m-r \geq 0 \\
m+r \leq P_{L} \\
S\left(T_{0}\right) \leq C \\
S\left(T_{0}\right)=S_{0}+\Delta t \sum_{j=1}^{l_{0}} x_{j} \\
T_{0}=l_{0} \Delta t \\
\frac{C-S\left(T_{0}\right)}{P_{L}} \leq T-T_{0}
\end{array}
$$

Note that the regulation signals $x_{j}$ 's are stochastic (unless we conservatively model them using the maximal and minimal deterministic regulation sequences as will be discussed later) and therefore, $S\left(T_{0}\right)$ is a random variable in the optimization problem 5. As a result, in general, we need to use the statistical properties of $S(t)$ when studying this optimization problem.

In the following, we will study two particular cases of interest. The first corresponds to the case where we assume that the cumulative sum of the regulation signals is Gaussian while the second is the deterministic worst case. 


\subsection{The Optimization Problem under the Gaussian Assumption}

\subsubsection{Formulation}

In a single-shot stochastic process, the values of $m$ and $r$ are not changed during the charging process. Thus, once the values of these parameters are set, the system must be ready to deal with the worst-case scenarios that may happen as a result of this selection. Let $\mu_{S}(t)$ and $\sigma_{S}^{2}(t)$ denote the mean and variance of $S(t)$ at time $t$. Also, let $P_{e}$ be the maximum tolerable error in the system. There are, a priori, two types of potential errors. The first corresponds to the situation when the system gets fully charged before the end of the regulation service period and cannot provide regulation service any more, and the second to the situation when the system is not fully charged by the end of the charging period. We design the system in such a way that with probability $1-P_{e}$ neither error occurs in the system. We assume that the cumulative sum of the regulation signals is Gaussian which translates into a Gaussian assumption on the system residual capacity. Let us fix the value of $P_{e}$. Under the Gaussian assumption, the value of $S(t)$ lies with probability 1- $P_{e}$ within the interval $\left[\mu_{S}(t)+\alpha \sigma_{S}(t), \mu_{S}(t)-\alpha \sigma_{S}(t)\right]$ where $\alpha=-G^{-1}\left(\frac{P_{e}}{2}\right)$, and $G(\cdot)$ is the normal standard distribution function. Using these notations, the system optimization problem, in its worst case (i.e., assuming the worst case trajectories for $S(t))$, can be expressed as: 


$$
\begin{array}{cc}
\max _{m, r, T_{0}, l_{0}} & r T_{0} \\
\text { s.t. } & m, r, T_{0} \geq 0, T_{0} \leq T \\
m-r \geq 0 \\
m+r \leq P_{L} \\
\mu_{S}\left(T_{0}\right)+\alpha \sigma_{S}\left(T_{0}\right) \leq C \\
T_{0}=l_{0} \Delta t \\
\frac{C-\left[\mu\left(T_{0}\right)-\alpha \sigma_{S}\left(T_{0}\right)\right]}{P_{L}} \leq T-T_{0}
\end{array}
$$

From Eq. 4, it can be shown that the mean of $S(t)$ at time $t=l \Delta t$ is given by (see Appendix A):

$$
\mu_{S}(t)=S_{0}+m l \Delta t=S_{0}+m t
$$

Also, it is not hard to show that the variance of $S(t)$ at time $t=l \Delta t$ is given by $\sigma_{S}^{2}(t)=r^{2} \sigma_{0}^{2}(t)$ where $\sigma_{0}^{2}(t)$ can be expressed in terms of the autocorrelation function of the stochastic process $v$ (recall that the regulation signal sequence is $x_{j}=m+r v_{j}$ for all $1 \leq j \leq k$ where $v$ denotes a zero-mean stochastic sequence in $[-1,1]$ ) as follows (Appendix A):

$$
\sigma_{0}^{2}(t)=t \Delta t\left(R_{v}(0)+2 \sum_{i=1}^{l-1} R_{v}(i)\right)-2(\Delta t)^{2} \sum_{i=1}^{l-1} i R_{v}(i)
$$


Here, $R_{v}(i)=\mathbb{E}\left[v_{j} v_{j-i}\right]$ denotes the autocorrelation function of $v$. Note that $\sigma_{0}^{2}(t)$ only depends on the basic regulation signal $v$, and not on parameter $r$. In the special case when the incoming regulation signals are uncorrelated, $R_{v}(i)=0$ for $i \geq 1$, and thus $\sigma_{0}^{2}(t)$ simplifies to:

$$
\sigma_{0}^{2}(t)=t \Delta t R_{v}(0)=t \Delta t \sigma_{v}^{2}
$$

where $\sigma_{v}^{2}=R_{v}(0)$ is the variance of the signal $v$.

In general, however, the signal $v$ is correlated and it is important to take these correlations into account.

Substituting values of $\mu_{S}(t)$ and $\sigma_{S}(t)$ from Eq. 7 and Eq. 8 in the optimization problem 6, we obtain:

$$
\begin{array}{cc}
\max _{m, r, T_{0}, l_{0}} & r T_{0} \\
\text { s.t. } & m, r, T_{0} \geq 0, T_{0} \leq T \\
m-r \geq 0 \\
m+r \leq P_{L} \\
S_{0}+m T_{0}+\alpha r \sigma_{0}\left(T_{0}\right) \leq C \\
T_{0}=l_{0} \Delta t \\
\frac{C-\left[S_{0}+m T_{0}-\alpha r \sigma_{0}\left(T_{0}\right)\right]}{P_{L}} \leq T-T_{0}
\end{array}
$$

In this problem, $T_{0}$ is an integer multiplier of $\Delta t$. In the following, we will 
relax this constraint when solving the optimization problem 10 and allow $T_{0}$ to take any real value within the interval $[0, T]$. This is a reasonable assumption, because $\Delta t$ is much smaller than other time scales in the problem.

This problem can be complex depending on the function $\sigma_{0}(t)$ which is a function of the autocorrelation function of $v$. Before providing analytical insights and solutions to this problem for different examples, we first provide some geometric interpretation.

\subsubsection{Geometric representation}

We find it useful to illustrate the constraints presented in 10 using a geometric representation. Such a geometric representation is given in Fig. 1. In this diagram, the vertical axis denotes the system residual capacity and the horizontal axis represents time. Each path within the presented rectangle illustrates a sample trajectory for charging the system based on given trajectories of regulation signals. All feasible trajectories must start from the bottom left corner of the rectangle, $\left(0, S_{0}\right)$, and end at the top right corner $(T, C)$. Because of the limitation on the peak power of the system, denoted by $P_{L}$, all feasible trajectories must lie within the two parallel lines with slope $P_{L}$, one crossing point $\left(0, S_{0}\right)$ and the other one point $(T, C)$. Consider a variable-rate regulation signal with mean $m$ and maximum deviation $r$. In this case, the feasible trajectories are limited by the two lines crossing point 
$\left(0, S_{0}\right)$ with slopes $m-r$ and $m+r$ respectively. Define:

$$
\begin{aligned}
& f_{m, r}^{\text {up }}(t)=\mu_{S}(t)+\alpha \sigma_{S}(t)=S_{0}+m t+\alpha r \sigma_{0}(t) \\
& f_{m, r}^{\text {low }}(t)=\mu_{S}(t)-\alpha \sigma_{S}(t)=S_{0}+m t-\alpha r \sigma_{0}(t)
\end{aligned}
$$

As mentioned in the previous subsection, all trajectories of the regulation signal lay between $f_{m, r}^{\text {up }}(t)$ and $f_{m, r}^{\text {low }}(t)$ with a probability that can be controlled by parameter $\alpha$. We denote by $T_{1}$ the intersection between the curve $f_{m, r}^{\text {up }}(t)$ and the horizontal line $y=C$ or the line $t=T$ whichever comes first. Also, we denote by $T_{2}$ the intersection between the curve $f_{m, r}^{\text {low }}(t)$ and the line with slope $P_{L}$ crossing point $(T, C)$ or the line $y=C$ whichever comes first. Given the values of $m$ and $r$, the optimal value of $T_{0}$ is the smaller of the two first coordinates of $T_{1}$ and $T_{2}$. That is because the largest value of $T_{0}$ that satisfies both constraints 11 and 12 is $\min \left(T_{1}, T_{2}\right)$.

\subsubsection{Solution to the optimization problem}

In this section, we reduce the nonlinear three variable optimization problem 10 to a simple one variable optimization problem over the interval $\left(T_{I}, T\right)$ (see later), which can be solved precisely. We start by presenting the following key theorem:

Theorem 2. Let $\left(m^{\mathrm{opt}}, r^{\mathrm{opt}}, T_{0}^{\mathrm{opt}}\right)$ be the optimal solution to problem 10 . At this optimal solution, both inequalities 11 and 12 are active (i.e., hold at equality) provided that $g(t)=\frac{\sigma_{0}(t)}{t}$ is a decreasing function of $t$. 
Proof. See appendix B.

The geometric interpretation of the above theorem is that at the optimal solution, the values of the first coordinates of $T_{1}$ and $T_{2}$ (in Fig. 1) are equal to each other and equal to $T_{0}$, provided that $g(t)=\frac{\sigma_{0}(t)}{t}$ is a decreasing function of $t$.

In the special case when the incoming regulation signals are uncorrelated, it can be seen that $g(t)=\frac{\sigma_{0}(t)}{t}=\frac{\sqrt{\sigma_{v}^{2} \Delta t}}{\sqrt{t}}$ is a decreasing function of $t$. Theorem 3 below indicates that the same result is indeed true for all signals with a positively-valued autocorrelation function $R_{v}(\cdot)$.

Theorem 3. $g(t)=\frac{\sigma_{0}(t)}{t}$ is a decreasing function of $t$ if the corresponding autocorrelation function $R_{v}$ is positively-valued.

Proof. See appendix C.

Examples of positively-valued autocorrelation functions include triangular and exponential autocorrelation functions. In Subsection 6.2.2, we obtain the autocorrelation function of real-world PJM Interconnection regulation traces. As we will see there, the autocorrelation function of these signals has a triangular form, and thus the above theorem can be applied.

Given Theorem 2, one can replace inequalities 11 and 12 in the optimization problem 10 by equalities. We can then evaluate the value of $m$ to obtain 
the following equation in terms of $r$ and $T_{0}$ :

$$
r=\frac{\left(T-T_{0}\right) P_{L}}{2 \alpha \sigma_{0}\left(T_{0}\right)}
$$

After some simple algebra, we arrive at the following optimization problem which includes only variables $m$ and $T_{0}$ :

$$
\begin{array}{cc}
\max _{m, T_{0}} & \frac{P_{L}}{2 \alpha} \frac{\left(T-T_{0}\right) T_{0}}{\sigma_{0}\left(T_{0}\right)} \\
\text { s.t. } & m, T \geq 0, T_{0} \leq T \\
& m-\frac{\left(T-T_{0}\right) P_{L}}{2 \alpha \sigma_{0}\left(T_{0}\right)} \geq 0 \\
& m+\frac{\left(T-T_{0}\right) P_{L}}{2 \alpha \sigma_{0}\left(T_{0}\right)} \leq P_{L} \\
& S_{0}+m T_{0}+\frac{\left(T-T_{0}\right) P_{L}}{2}=C
\end{array}
$$

To gain insights on the above optimization problem, it is useful to plot constraints 15, 16 and 17 in the $m-T_{0}$ plane (see Fig. 22). To do so, we first note that inequality [15] corresponds to the area above the curve $C_{1}$ defined by:

$$
m=\frac{\left(T-T_{0}\right) P_{L}}{2 \alpha \sigma_{0}\left(T_{0}\right)}
$$

Similarly, we note that the inequality presented in 16 corresponds to the 
area below the curve $C_{2}$ defined by:

$$
m=-\frac{\left(T-T_{0}\right) P_{L}}{2 \alpha \sigma_{0}\left(T_{0}\right)}+P_{L}
$$

At $T_{0}=T$, the curves $C_{1}$ and $C_{2}$ take the value 0 and $P_{L}$, respectively. Moreover, it can be seen that these curves are symmetric around the line $m=P_{L} / 2$ in the $m-T_{0}$ plane, and thus always intersect at a point with height $P_{L} / 2$ (Fig. 2).

Finally, let the curve represented by Eq. 17 be $C_{3}$. We rewrite its equation as:

$$
m=\frac{\frac{C-S_{0}}{T}-\frac{P_{L}}{2}\left(1-\frac{T_{0}}{T}\right)}{T_{0} / T}
$$

Let $P_{C}=\frac{C-S_{0}}{T}$. Note that this quantity represents the average power required to fill all the batteries by the end of the charging period. To study the behavior of the system, we find it useful to define the power ratio $Q$ as follows:

$$
Q=\frac{P_{C}}{P_{L} / 2}
$$

In plotting the curve $C_{3}$, we distinguish between the following three regimes: (i) $Q<1$ (or $P_{C}<\frac{P_{L}}{2}$ ), (ii) $Q>1$ (or $P_{C}>\frac{P_{L}}{2}$ ) and (iii) $Q=1$ (or $P_{C}=\frac{P_{L}}{2}$ ). One can see that the convexity of this curve depends on the regime in which the system operates. The feasible solutions for the optimization problem 14 are those points on $C_{3}$ that lay above $C_{1}$ and below 
$C_{2}$.

After finding the feasible region, we turn our attention to the objective function in the optimization problem 14. The objective function in this problem depends only on $T_{0}$. From Fig. 2 it can be seen that the feasible values for $T_{0}$ lay in the interval $\left(T_{I}, T\right)$, where $T_{I}$ is the first coordinate of the intersection between curves $C_{3}$ and $C_{1}$ or $C_{2}$ (depending on the regime in which the system is operating). Therefore, to solve the optimization problem 14, one needs to find the value of $T_{0}$ in the interval $\left(T_{I}, T\right)$ that maximizes the objective function. Our computations shows that in many practical cases the objective function 14 is strictly decreasing over the interval $\left(T_{I}, T\right)$ and thus the optimal value of $T_{0}$ will be the starting point of this interval, i.e., $T_{I}$.

A special case: Uncorrelated regulation signals. We now study the solution to 14 in a special case when the incoming regulation signals are uncorrelated. As we will see here, the solution in this case can be obtained by solving a third-order polynomial equation.

As discussed earlier, in the case of uncorrelated regulation signals $\sigma_{0}^{2}(t)=$ $t \Delta t \sigma_{v}^{2}$. With this value for $\sigma_{0}^{2}(t)$, the curves $C_{1}$ and $C_{2}$ intersect at $\left(T_{J}, P_{L} / 2\right)$, where:

$$
T_{J}=\frac{(\sqrt{4 T+\kappa \Delta t}-\sqrt{\kappa \Delta t})^{2}}{4}
$$

Here $\kappa=\left(\alpha \sigma_{v}\right)^{2}$ is a constant. Note that in practical systems where 
$\Delta t \ll T$ the value of $T_{J}$ in 22 will be close to $T$.

Next we study the objective function in 14 for this special case. Using the above value for $\sigma_{0}^{2}(t)$ the objective function is:

$$
f\left(T_{0}\right)=\frac{P_{L}}{2 \alpha \sigma_{v} \sqrt{\Delta t}}\left(T-T_{0}\right) \sqrt{T_{0}}
$$

It is easy to see that the above function has a global maximum at $T_{0}=$ $T / 3$, and it is decreasing for values of $T_{0}$ greater than $T / 3$. As mentioned earlier, to obtain the optimal solution to the optimization problem 14, we must find the value of $T_{0}$ in the interval $\left(T_{I}, T\right)$ that maximizes the objective function. Recall that $T_{I}$ is the first coordinate of the intersection between curves $C_{3}$ and one of $C_{1}$ or $C_{2}$ depending on the regime in which the system is operating. From Fig. 2, it can be seen that in all regimes the value of $T_{I}$ is greater than or equal to $T_{J}$. As mentioned above, in practical systems the value of $T_{J}$ is close to $T$, and since $T_{J} \leq T_{I}<T$, the value of $T_{I}$ will be close to $T$ too. As a result, the whole interval $\left(T_{I}, T\right)$ lays on the right-hand side of the point $T_{0}=T / 3$, and the objective function 23 is strictly decreasing over the interval $\left(T_{I}, T\right)$. This implies that the optimal value of $T_{0}$ will be the starting point of the interval $\left(T_{I}, T\right)$, i.e., $T_{I}$.

As mentioned in the previous subsection, when $Q<1$ (the first regime), the value of $T_{I}$ is given by the first coordinate of the intersection between curves $C_{1}$ and $C_{3}$. Using simple algebra, we obtain the optimal value of $T_{0}$ as $x^{2}$, where $x$ is the solution to the following cubic polynomial: 


$$
\begin{aligned}
& a x^{3}+b x^{2}+c x+d=0, \\
& a=\frac{P_{L}}{2 \alpha \sigma_{v} \sqrt{\Delta t}} \\
& b=\frac{P_{L}}{2} \\
& c=\frac{-P_{L} T}{2 \alpha \sigma_{v} \sqrt{\Delta t}} \\
& d=C-S_{0}-\frac{P_{L} T}{2}
\end{aligned}
$$

Similarly, in the second regime where $Q>1$, it can be seen that the optimal value of $T_{0}$ is given by $y^{2}$ where $y$ is the solution to the cubic polynomial:

$$
a y^{3}+b y^{2}+c y-d=0
$$

Once we obtained the optimal value of $T_{0}$ for these 2 regimes, we can obtain the optimal values of $m$ using Eq. 20, and the optimal value of $r$ using Eq. 13,

Finally, in the third regime where $Q=1$, the three curves $C_{1}, C_{2}$ and $C_{3}$ all intersect at $\left(T_{J}, P_{L} / 2\right)$. It can be seen that in this case the optimal value of $r$ equals $\frac{P_{L}}{2}=m$.

In this section, therefore, we have reduced the problem to a simple singlevariable optimization problem. 


\subsection{Optimal solution in the deterministic case}

In this subsection, we study the solution to optimization problem 5 in the (deterministic) worst case where regulation signals are all equal to each other, and equal to either $m+r$ or $m-r$, corresponding to the maximal and minimal regulation sequence, respectively. We find closed form solutions for this problem in all regimes (i.e., for all values of $Q$ ). The solution in this case provides a measure of the degree of improvement that can be obtained by taking a stochastic approach to the problem. This setting is illustrated by the two lines crossing point $\left(0, S_{0}\right)$ with slopes $m+r$ and $m-r$ in Fig. 1. We use the same definition for $T_{1}$ and $T_{2}$ as before when we replace the curve $f_{m, r}^{\text {up }}(t)$ (resp. $\left.f_{m, r}^{\text {low }}(t)\right)$ by the line of slope $m+r(\operatorname{resp} m-r)$. With these definitions, the value of $T_{0}$ will be again $\min \left\{T_{1}, T_{2}\right\}$. One can follow a procedure similar to the one described in Appendix B to obtain the optimal values of $m, r$ and $T_{0}$ in this deterministic setting. Theorem 4 below gives the solutions in this case.

Theorem 4. Consider the optimization problem 5 in the deterministic case where regulation signals are all equal to each other, and equal to either $m+r$ or $m-r$.

- When $Q<1$, any value in the interval $\left(P_{1}, P_{L} / 2\right)$ is optimal for $m$, where:

$$
P_{1}=\frac{P_{C}}{2} \frac{1}{1-\frac{P_{C}}{P_{L}}}
$$

In this case, the optimal value of $r$ is $m$, and the optimal value of the 
objective function $r T_{0}$ is $\frac{C-S_{0}}{2}$.

- When $Q \geq 1$, the optimal values of $m$ lay in the interval $\left[\frac{P_{L}}{2}, P_{2}\right]$, where:

$$
P_{2}=\frac{P_{L}}{2} \frac{3 \frac{P_{C}}{P_{L}}-1}{\frac{P_{C}}{P_{L}}}
$$

In this case, the optimal value of $r$ is equal to $P_{L}-m$, and the optimal value of the objective function $r T_{0}$ is $\frac{P_{L} T-C+S_{0}}{2}$.

Proof. See Appendix D.

As mentioned in the above theorem, the optimal solutions in the deterministic case are not unique. Moreover, one can see that the values $P_{L} / 2$ and $P_{C}$ always lay in the interval of optimal values for $m$.

\subsection{Results in the case of a representative system}

In this subsection, we illustrate some of the results obtained in the previous subsections for a representative system. We consider a fleet of electric vehicle consisting of 80 vehicles, each with a $20 \mathrm{kWh}$ storage unit. We assume the fleet is available for charging from 10 PM to 6 AM; thus, the total charging period is 8 hours. Using the notations introduced in the previous subsection, we have $n=80, C_{s}=20 \mathrm{kWh}$ and $T=8 \mathrm{~h}$.

If all vehicle batteries are empty at the beginning of the charging period, the system needs a total energy of $80 \times 20=1600 \mathrm{kWh}$ to get fully charged by the next day's morning. Given the length of the charging period, 8 hours, the input peak power is expected to be $P_{L} \geq 1600 \mathrm{kWh} / 8 \mathrm{~h}=200 \mathrm{~kW}$. Because 
we plan to provide regulation service, the input peak power should be chosen greater than $200 \mathrm{~kW}$. Larger values of the input peak power are expected to result in higher values of regulation service. We will study the effect of input peak power on the optimal value of regulation service later in this subsection. We assume that the autocorrelation function of the regulation signals has a triangular shape. As we will see in Section 6.2, this is consistent with the results obtained from real-world data. We define the correlation time $T_{C}$ of a triangular autocorrelation function as the time-lag beyond which the autocorrelation function vanishes, i.e., the time-lag beyond which the incoming regulation signals are uncorrelated. Based on the results obtained from the real-world data (see subsection 6.2.2), we set $T_{C}=45 \mathrm{~min}$ and $\sigma_{v}=0.5$ (unitless). We take the probability of error in the system to be $P_{e}=10^{-3}$. Finally, we assume the batteries of the vehicles are initially charged at $25 \%$ on average. This leads the system initial residual capacity to be $25 \% \times 80 \times 20 \mathrm{kWh}=400 \mathrm{kWh}$, and the required average power, $P_{C}$, to be:

$$
P_{C}=\frac{C-S_{0}}{T}=\frac{1600 \mathrm{kWh}-400 \mathrm{kWh}}{8 \mathrm{~h}}=150 \mathrm{~kW}
$$

Figure 3 plots the value of the regulation service $r T_{0}$ as a function of the input peak power $P_{L}$. In plotting Fig. 3, we keep $P_{C}$ constant at $150 \mathrm{~kW}$ and vary $P_{L}$ in the range $200-400 \mathrm{~kW}$. We find it useful to normalize the horizontal axis and plot the value of regulation service as a function of the 
power ratio $Q$ defined in 21. Figure 3 compares the optimal value of the regulation service obtained when assuming a Gaussian cumulative sum of the regulation signals with a triangular autocorrelation function with the value of the regulation service obtained from the following four sub-optimal methods:

- Stochastic with $m=P_{C}$. In this method, the mean charge rate $m$ is taken equal to $P_{C}$, and the value of $r$ is then equal to $\min \left\{P_{C}, P_{L}-P_{C}\right\}$. Given the values of $m$ and $r$, the functions $f_{m, r}^{\text {up }}(t)$ and $f_{m, r}^{\text {low }}(t)$ can be evaluated, and from those, values of $T_{1}$ and $T_{2}$ are obtained. The value of $T_{0}$ is then equal to $\min \left\{T_{1}, T_{2}\right\}$.

- Stochastic with $m=\frac{P_{L}}{2}$. In this method, the mean charge rate $m$ and deviation parameter $r$ are both taken equal to $\frac{P_{L}}{2}$. As in the previous case, given the values of $m$ and $r$, the functions $f_{m, r}^{\text {up }}(t)$ and $f_{m, r}^{\text {low }}(t)$ are evaluated, and from those, values of $T_{1}$ and $T_{2}$ are obtained. The value of $T_{0}$ is then equal to $\min \left\{T_{1}, T_{2}\right\}$.

- Deterministic with $m=P_{C}$. In this method, similar to the first case above, the mean charge rate $m$ is set to $P_{C}$ and we allow for deviations of at most $r=\min \left\{P_{C}, P_{L}-P_{C}\right\}$ above or below this mean but, we account for the worst cases where all regulation signals are equal to each other and equal to either $P_{C}+r$ or $P_{C}-r$.

- Deterministic with $m=\frac{P_{L}}{2}$. In this method, similar to the second case above, the mean charge rate $m$ and the deviation parameter $r$ are 
both equal to $P_{L} / 2$ but again we account for the cases where all signals can be equal to each other and equal to either $P_{L}$ or 0 . Note that Theorem 4 predicts that the value of the regulation service obtained from this method and the previous one are both optimal in a deterministic regime and equal to each other.

As illustrated in Fig. 3, the amount of regulation service provided by taking into account the stochastic nature of the signals is, in general, greater than the value of the regulation service provided by the deterministic approaches. Moreover, not surprisingly, the values of regulation service for the two deterministic case are equal to each other. As illustrated in Fig. 3, $Q=1$ yields nearly the highest regulation service. This implies that the utility should choose to design the system so that $P_{L}$ is at least equal to $2 \frac{C-S_{0}}{T}$. We will discuss this issue later in the present section.

Next, we study the effect of the system initial residual capacity $S_{0}$ on the optimal value of the regulation service. To this end, we keep the input peak power constant at $P_{L}=300 \mathrm{kWh}$, and vary the system initial residual capacity between $0 \%$ and $50 \%$. The results are presented in Fig. 4 as a function of $Q=\frac{2\left(C-S_{0}\right)}{T P_{L}}$. Figure 4(a) plots the optimal value of the regulation service as a function of the power ratio $Q$. In this figure, we have compared the proposed optimal method with the four sub-optimal methods mentioned above. Figure $4(\mathrm{~b})$ compares the length of the regulation period $T_{0}$ for the same methods. As illustrated in Fig. 4(a), given the values of $P_{L}$ and $T$, the optimal value of regulation service is maximized when $Q$ turns out to be one, 
or equivalently, the initial residual capacity of the system turns out to be $C-$ $\frac{T P_{L}}{2}$. From Fig. $4(\mathrm{~b})$ it is clear that the system with the optimal parameters provides, in general, longer durations of regulation service. Importantly, it can be seen from Figs. 3 and 4 (a) that the value of the regulation service obtained from the optimal method, and the (suboptimal) stochastic method with $m=P_{C}$ are close to each other in all three cases $Q<1, Q=1$ and $Q>1$. This implies that in practical applications, the value of $m=P_{C}$ can be used as a good approximation to the optimal value of $m$.

In Fig. 5, we study the effect of the correlation time $T_{C}$ of the incoming regulation signals on the optimal value of the regulation service. In plotting this figure, the values of $P_{C}$ and $P_{L}$ are kept constant at $150 \mathrm{~kW}$ and 300 $\mathrm{kW}$, respectively, and the correlation time between the regulation signals is varied between 0 and 2 hours. As shown in this figure, the optimal value of the regulation service decreases as the correlation among the regulation signals increases. This is because when regulation signals are not correlated, or are correlated with small correlation times, regulation up and regulation down signals cancel the effect of each other over short periods of time, and the energy sent to the fleet remains close to its expected value. However, when regulation signals are correlated over longer intervals, it may be the case that a sequence of consecutive up or down regulation signals appear in the system, and lead the system residual capacity to largely deviate from its expected value. In such situations, the variance of the system residual capacity increases, and the value of the regulation service decreases for a 
given probability of error. In Fig. 5, we have only presented values of the regulation service greater than or equal to $600 \mathrm{~kW}-\mathrm{h}$. That is because, as shown in Fig. 4(a), the deterministic approach that achieves zero error can yield a regulation service value of $600 \mathrm{~kW}$-h. All other proposed methods are useful only if they offer regulation service values greater than this errorfree case. This illustrates that depending on the values of the parameters, a deterministic approach can be the best solution. In this figure, we also show how the choice of the error probability impacts the optimal regulation service. Clearly the higher the probability, the higher the regulation service.

Finally, in Fig. 6 we study the effect of the variance of the base signal, $\sigma_{v}$, on the optimal value of the regulation service for different values of the error probability. As shown, the optimal value of the regulation service decreases quickly as $\sigma_{v}$ increases. That is because, the variance of the system residual capacity, $S(t)$, increases as $\sigma_{v}$ increases (see Eq. 8), and the resulting increased uncertainty leads to more conservative estimates of the value of the regulation service achievable in the system. As in Fig. 5, the diagram in Fig. 6 presents only values of the regulation service greater than or equal to 600 $\mathrm{kW}-\mathrm{h}$.

\subsection{Setting the physical parameters $P_{L}$ and $p$}

In this section, we study how to set parameters $P_{L}$ and $p$ in a system of electric vehicles that is planned to provide the regulation service. This is a one-time parameter design and should be performed when the infrastructure 
of the fleet is designed. We denote the obtained values of parameters by $P_{L}^{\text {des }}$ and $p^{\text {des }}$, respectively.

As mentioned in Section 5, in order to get a good value for the regulation service, we should have $Q \leq 1$ irrespective of the value of the initial residual capacity $S_{0}$. Hence, the value of $P_{L}$ should be set to be twice as large as the value of $P_{C}$. Larger values of $P_{L}$ increase the price of the connection line, but they do not significantly increase the value of regulation service (see Fig. 3). The maximum value of $P_{C}$ corresponds to the case when all storage units are fully discharged at the beginning of the charging process. In that case, the value of $P_{C}$ equals to $\frac{n C}{T}$. To ensure that the full potential of the system is used for providing the regulation service (irrespective of the value of $S_{0}$ ), $P_{L}^{\text {des }}$ should be set based on this worst-case scenarid 3 :

$$
P_{L}^{\text {des }}=2 \frac{n C}{T}
$$

Now we study how to set the value of $p$. As mentioned in Section 4 , the performance of a distributed storage system is upper bounded by a single big storage unit. This upper bound can be achieved if the value of $p$ is sufficiently large. The minimum value of $p$ that achieves this upper bound can be obtained using condition 1. Hence:

\footnotetext{
${ }^{3}$ Alternatively, if an accurate estimate of $P_{C}$ is available, one may use this value to determine $P_{L}^{\text {des }}$.
} 


$$
\begin{aligned}
p^{\text {des }} & \geq \frac{P_{L}^{\text {des }}}{\sum_{i=1}^{n} R_{i}} R_{\max } \\
& =\frac{2 \frac{n C}{T}}{\sum_{i=1}^{n} R_{i}} R_{\max } \\
& =\frac{2}{\frac{\sum_{i=1}^{n}\left(R_{i} / C\right)}{n}} \frac{R_{\max }}{T} \\
& =\frac{2}{1-\frac{\sum_{i=1}^{n}\left(S_{i} / C\right)}{n}} \frac{R_{\max }}{T} \\
& =\frac{2}{1-\beta} \frac{R_{\max }}{T}
\end{aligned}
$$

where $\beta=\frac{\sum_{i=1}^{n}\left(S_{i} / C\right)}{n}$ is the average normalized initial residual capacity of the individual units. A conservative value for $p^{\text {des }}$ that ensures the validity of 26 can be obtained by replacing $R_{\max }$ by $C$ in this inequality. This results in the following lower bound for $p^{\text {des: }}$

$$
p^{\mathrm{des}} \geq \frac{2}{1-\beta} \frac{C}{T}
$$

Hence, the value of $p^{\text {des }}$ depends on the average residual capacity of the individual storage units. Although the residual capacity of the storage units may vary from day to day, their average may have less variations. Given an estimate for the value of $\beta$ in the system we can use inequality 27 to obtain the minimal value of $p^{\text {des }}$. From this inequality, it can be seen that if the value of $\beta$ varies between $0 \%-50 \%$, the minimal value of $p^{\text {des }}$ varies between 
$\frac{2 C}{T}$ and $\frac{4 C}{T} .4$

We are now ready to evaluate the values of parameters $P_{L}^{\text {des }}$ and $p^{\text {des }}$ for the representative system introduced in Section 5.3, Using Eq. 25, the input peak power in this system should be set at $P_{L}^{\text {des }}=2 \frac{n C}{T}=2 \frac{80 \times 20 \mathrm{kWh}}{8 \mathrm{~h}}=$ $400 \mathrm{~kW}$. Moreover, assuming an average initial residual capacity of 25\%, the individual peak powers should be set to a minimum of $p^{\text {des }}=\frac{2}{1-\alpha} \frac{C}{T}=$ $\frac{2}{1-0.25} \frac{20 \mathrm{kWh}}{8 \mathrm{~h}}=6.66 \mathrm{~kW}$. This line power can be achieved by a level 2 charging infrastructure [5].

We conclude this section by considering the case when condition 1 does not hold, e.g., when the initial residual capacity of the storage units is relatively high ( small $R$ ) and this initial charge is distributed heterogeneously among the individual units (large $R_{\max }$ ). In such cases, one can replace the input peak power $P_{L}$ by a modified value that can be obtained from condition 1, as follows:

$$
P_{L}^{\mathrm{mod}}=\frac{\sum_{i=1}^{n} R_{i}}{R_{1}} p
$$

This modified value of $P_{L}$ can replace the value of $P_{L}$ in Section 5 to determine the charging parameters of the system.

\footnotetext{
${ }^{4}$ One should note that if the value of $\beta$ approaches 1 , the right-hand side of inequality 27 goes to infinity. In such cases, the original condition in 26 should be used to obtain the value of $p^{\text {des }}$ which includes both $\beta$ and $R_{\max }$.
} 


\section{Dynamic Optimization}

\subsection{Introduction}

In the single-shot optimization studied in Section 5, the optimal values of $m$ and $r$ are set at the beginning of the charging process and will not change until the end of the regulation service period. In this system, because of the one-shot nature of the process, the parameter values should be chosen conservatively so as to ensure the successful operation of the system. One can extend the above system to a dynamic setting in which the values of $m$ and $r$ are updated multiple times during the charging process. The updated values of $m$ and $r$ will be then determined based on the current state of the system at each updating point. We expect this dynamic setting to improve the total value of the regulation service provided by the system. However, we should note that the time span between updating points in a dynamic setting cannot be too short. Otherwise, frequently altering the system parameters (including the requested mean power) may serve as a source of fluctuations in the grid, rather than a mean of absorption of the fluctuations generated by other sources.

In this section, we introduce a simple dynamic setting for charging the fleet of electric vehicles. In particular, we study a system in which the values of $m$ and $r$ are updated $d+1$ times at points $t=0, T_{u}, 2 T_{u}, \cdots, d T_{u}$. At this point, we assume that the value of $T_{u}$ is given and that the value of $d$ will be determined during the charging period, as will be described shortly. We examine the system at each updating point, and evaluate the three param- 
eters $m, r$ and $T_{0}$ based on the current residual capacity of the system and the time remaining until the end of the charging period $T$. Let $m^{i}, r^{i}$ and $T_{0}^{i}$ denote, respectively, the values of $m, r$ and $T_{0}$ at updating point $t_{i}=i T_{u}$. If the value $T_{0}^{i}$ is greater than $T_{u}$, it means that the system can safely work until the next update point $t_{i+1}=(i+1) T_{u}$. In that case, we continue providing the regulation service until time $t_{i+1}=(i+1) T_{u}$, and again check the system at time $t_{i+1}$. On the other hand, if the value of $T_{0}^{i}$ evaluated at the updating point $t_{i}$ is less than $T_{u}$, we provide regulation service until $t_{i}+T_{0}^{i}$ and then switch to a deterministic phase to finish the charging process by time $T$, i.e., in that case, $d=i$. Figure 7 illustrates the geometric representation of a typical system with dynamic charging.

Let $T_{\text {reg }}^{i}$ denote the length of the $i^{\text {th }}$ regulation period during the charging process described above. Note that $T_{\mathrm{reg}}^{0}=T_{\mathrm{reg}}^{1}=\cdots=T_{\mathrm{reg}}^{d-1}=T_{u}$ and $T_{\text {reg }}^{d}=T_{0}^{d}$. The total value of the regulation service provided by the system can be then expressed as:

$$
\text { Equivalent service }=\sum_{i=0}^{d} r^{i} T_{\text {reg }}^{i}
$$

We study the dynamic setting in both the deterministic and the stochastic cases. In the deterministic case, as mentioned in Section 5 , there are a range of optimal values for the mean charge parameter $m$. We study two special cases where $m$ is set to $\frac{P_{L}}{2}$ and to $P_{C}$. The advantage of choosing $\frac{P_{L}}{2}$ over $P_{C}$ is that this choice makes the system parameters independent of the residual 
capacity at each updating point.

We will see next that the value of the regulation service will significantly increase in a system with dynamic setting, and that the difference between the deterministic and the stochastic settings reduces.

\subsection{Numerical Evaluations}

In this section, we use simulation and real-world regulation data to obtain results for different settings. To this end, we use the representative system described in Section 5.3, Sections 6.2.1 and 6.2.2 use simulation and realworld data, respectively, to assess the performance of the system. In each of these subsections, we study both single-shot and dynamic settings.

\subsubsection{Simulation data}

A) Single-shot optimization. In this subsection, we use simulation data to assess the value of the regulation service in a system using a single-shot optimization scheme. To this end, we study the representative system introduced in Section 5.1.3. We assume the system is initially 25\% charged. Moreover, we set $P_{L}=300 \mathrm{~kW}, T_{C}=45 \mathrm{~min}, \sigma_{v}=0.5$, and $P_{e}=10^{-3}$. Using the methodology presented in Section 5.1.3, we can show that the optimal values of the variables in the stochastic setting are $m^{\mathrm{opt}}=150 \mathrm{~kW}, r^{\mathrm{opt}}=150 \mathrm{~kW}$ and $T_{0}^{\mathrm{opt}}=4.92 \mathrm{~h}$. Given $r^{\mathrm{opt}}$ and $T_{0}^{\mathrm{opt}}$, the optimal value of the regulation service is $r^{\mathrm{opt}} T_{0}^{\mathrm{opt}}=738.1 \mathrm{~kW}-\mathrm{h}$.

To evaluate the system, we manually generate 100 zero-mean random traces with $T_{C}=45 \mathrm{~min}, \sigma_{v}=0.5$ and maximum and minimum +1 and -1 , 
respectively. Then, we multiply these traces by $r^{\text {opt }}$ and lift them up by $m^{\text {opt }}$ to obtain final regulation signal trajectories. We use these signals to charge the system of electric vehicles for a time duration with length $T_{0}^{\text {opt }}$. We seek to determine whether the regulation signals can be safely absorbed by the system during this interval. Moreover, we wish to determine whether the residual capacity of the system at time $t=T_{0}^{\text {opt }}$ allows the system to be fully charged by time $T$. We found that the generated trajectories almost always, up to the predicted error, lay within the $f_{m, r}^{\text {up }}$ and $f_{m, r}^{\text {low }}$ boundaries, and the regulation service was safely provided over the interval $\left[0, T_{0}\right]$. Moreover, residual capacity of the system at $t=T_{0}^{\text {opt }}$ was always large enough to allow the system to be fully charged by time $t=T$ using an input power less than $P_{L}$. These results confirm our predictions in Section 5.

From Theorem 4 it is easy to see that the optimal value of regulation service in the deterministic setting is $600 \mathrm{~kW}-\mathrm{h}$. This value is realized by $m^{\mathrm{opt}}=r^{\mathrm{opt}}=150 \mathrm{~kW}$ and $T_{0}^{\mathrm{opt}}=4 \mathrm{~h}$.

B) Dynamic optimization. In this subsection, we study how the value of regulation service increases by using dynamic optimization. Figure 8 shows the expected value of the regulation service as a function of time span between updates in the stochastic setting and in the deterministic setting with both $m=\frac{P_{L}}{2}$ and $m=P_{C}$. The system under study is the same system described in the previous section. In plotting Fig. 8, we generated 1000 random trajectories for $v$ (recall that $v$ is a zero-mean random sequence with $T_{C}=45 \mathrm{~min}$, $\sigma_{v}=0.5$ and maximum and minimum +1 and -1 , respectively), and use them 
to charge the system while the value of $T_{u}$ was varied between 0 and $T$. As illustrated in this figure, the resulting functions has a sawtooth shape. When the number of updates increases, the value of the regulation service increases sharply and, perhaps surprisingly, the deterministic setting outperforms the stochastic one. What is also surprising is that the two deterministic settings do not perform identically when the number of updates increases. As illustrated in the figure, even a single additional update can greatly improve the value of the provided regulation service.

\subsubsection{Real-world data}

In this section, we use regulation traces collected in 12 consecutive days by the PJM Interconnection company to study the system of electric vehicles.

This data is available at [13]. The regulation signals in this data set are normalized such that they lay within the range \pm 1 . We randomly divided the data into a training set consisting of four days, and a test set consisting of eight days. Figure 9 shows the QQ plot of the regulation traces in the training set versus standard normal. It shows that the regulation signals well match the distribution of a normal random variable truncated at \pm 1 . We evaluated the variance of the signals in the unified data stream to obtain an estimate for the value of $\sigma_{v}$. The obtained estimate for $\sigma_{v}$ was 0.5069 .

We then use the autocorrelation function of the regulation signals to estimate the correlation time of the regulation traces. Figure 10 shows the autocorrelation functions of the regulation data plotted for the four days in 
the training set. As illustrated in this figure, all autocorrelation functions exhibit a triangle-like shape. As described in Subsection 5.3, we define the correlation time of a triangular autocorrelation function as the lag time beyond which the autocorrelation function becomes zero. As shown in Fig. 10, the correlation time $T_{C}$ of the regulation signals is approximately equal to 45 min in all training days. We use the values of $\sigma_{v}=0.5069$ and $T_{C}=45 \mathrm{~min}$ in the remaining of this section. We also note that the mean of the regulation signals in the training set was equal to $\mu_{v}=-0.10$ instead of zero. To account for this difference, we lift the regulation signals in the test data by the mean value $\mu_{v}$ obtained from the training set. This change leads the base regulation signal to lay within the interval $[-0.9,1.1]$ rather than $[-1,1]$. We noted that this change did not affect the proper operation of the system. In general, it is interesting to extend the model presented here to the settings where the base signal $v(t)$ can have a nonzero mean.

A) Single-shot optimization. In this subsection, we use the real-world regulation traces described above to assess the value of the regulation service in a system using single-shot optimization. To study the system in the stochastic setting, we use the parameters $\sigma_{v}$ and $T_{C}$ obtained from the training set to solve the optimization problem for the the previously described system of electric vehicles. As before, we assume that the system is initially $25 \%$ charged, and the tolerable error in the system is $P_{e}=10^{-3}$. With these parameters we obtain $m^{\mathrm{opt}}=r^{\mathrm{opt}}=150 \mathrm{~kW}$ and $T_{0}^{\mathrm{opt}}=4.89 \mathrm{~h}$, leading to an optimal regulation service value of $r^{\text {opt }} T_{0}^{\text {opt }}=733.36 \mathrm{~kW}-\mathrm{h}$. 
Next, we multiplied the traces in the test set by $r^{\text {opt }}$ and lifted them by $m^{\text {opt }}$ to obtain trajectories for charging the system. The test set consisted of eight days. For two days in this set, the regulation data was available for only part of the day. We divided the regulation traces in each test day into blocks of approximately 8 hours. This data taken together provided a total of 22 blocks of regulation traces. We used these traces to charge the system for a period of $T_{0}^{\mathrm{opt}}=4.89$ hours, and then switched to a deterministic phase to completed the charging process by the end of the charging period at $T=8 \mathrm{~h}$. Figure 11 shows the geometric representation of the charging process in the system. As presented, the regulation service is successfully provided over the interval $\left[0, T_{0}^{\mathrm{opt}}\right]$ and there is always enough time for the system to get fully charged by the end of the charging period.

The optimal values of the regulation service and the the regulation service time in the deterministic setting are independent of the parameters $\sigma_{v}$ and $T_{C}$, and remain at $r^{\mathrm{opt}} T_{0}^{\mathrm{opt}}=600 \mathrm{~kW}-\mathrm{h}$ and $T_{0}^{\mathrm{opt}}=4 \mathrm{~h}$, respectively.

B) Dynamic optimization. In this subsection, we use the PJM regulation traces to study the fleet of vehicles in a dynamic setting. To this end, we again use the 22 blocks of regulation data obtained from the test data to assess the value of the regulation service. We consider both stochastic and deterministic settings.

Remember from the previous subsection that an appropriate value for $T_{u}$ in a dynamic setting can be a time duration slightly less than $T_{0}^{\text {opt }}$ in the single-shot setting. Therefore, we set the value of $T_{u}$ to $4.88 \mathrm{~h}$ in the stochas- 
tic, and to $3.99 \mathrm{~h}$ in the deterministic setting. Note that these are slightly less than the values of $T_{0}^{\text {opt }}$ obtained above for the corresponding single-shot settings. These values of $T_{u}$ achieve much of the improvement in the value of regulation service that is obtainable from the dynamic optimization while adding only a single updating point to the system. In the deterministic setting, we used $m=\frac{P_{L}}{2}$ at each update point. However, as illustrated in Fig.

8, the two choices of $m=\frac{P_{L}}{2}$ and $m=P_{C}$ are expected to perform similarly on average when $T_{u}$ is close to $T_{0}^{\mathrm{opt}}$. We noted that the average values of the regulation service value in the stochastic and deterministic settings were improved to $913.9 \mathrm{~kW}-\mathrm{h}$ and $848.9 \mathrm{~kW}$-h, respectively. As suggested by these numbers, the value of the regulation service can considerably increase by using the dynamic optimization, and the difference between the stochastic and the deterministic settings reduces in the dynamic setting. Figure 12 shows a geometric representation of the charging process in the stochastic setting.

\section{Conclusion and Future Work}

In this paper, we studied how a fleet of electric vehicles can provide frequency regulation service while getting charged overnight. Examples of potential fleets that can be used for this purpose include the DHL fleet of vehicles and the fleet of city transit buses. We studied a scenario in which the fleet is charged by a variable-rate signal overnight. In this scenario, we found the optimal values of the average charge rate and the maximum allowed deviation from the average. Our objective function was to maximize 
the value of the regulation service that can be offered to the grid.

Our results on a representative system indicate that if $P_{L}$ (the peak power of the fleet main line) is at least twice as large as $P_{C}$, the system can achieve much of the achievable regulation service value (Fig. 3). Also, we observed that the optimal value of the average power that should be requested from the grid $m$ is close to $P_{C}$ in all three regimes (Figs. 3 and 4 a). This implies that $P_{C}$ can be used as a good approximation to $m^{\text {opt }}$, in practice. We also observe that the optimal value of the regulation service in the system is inversely related to the correlation time of the regulation signals (Fig. 5). This is expected; when the correlation time of regulation signals is large, regulation up and down signals slowly compensates the effects of each other, and this leads to more conservative estimates of the regulation service.

We studied the relation between a distributed storage system and a single large storage unit. We obtained conditions under which the distributed system can be modeled as a single large storage unit, thus achieving its upper bound performance.

We studied dynamic optimization settings in which the value of the system parameters $m$ and $r$ are updated multiple times during the charging period. We observe that such settings can significantly increase the value of the regulation service provided by the system.

In addition to a stochastic setting, we studied the system in a worst case (deterministic) setting where regulation signals are all equal to each other, and equal to the maximal or minimal regulation sequence. The advantage of 
this system is that there is no risk attached to it, i.e., regulation can always be provided. We compared the regulation service obtained from the stochastic and deterministic settings in both single-shot and dynamic optimizations. We noted that although stochastic setting is expected to result in higher values of the regulation service, this difference in performance reduces with dynamic optimization. This implies that even without knowing the stochastics of the system, one can obtain high values of regulation service using a dynamic deterministic scheme.

The results presented in this work can be extended in multiple directions: First, we can study the optimal value of the regulation service when the equivalence condition between the distributed storage system and the single large storage unit (condition 1) does not hold. In this situation, we discussed replacing $P_{L}$ by a modified value provided in Eq. 28, However, this is not guaranteed to provide the optimal value of the regulation service. One should note that although it might be possible to improve the value of the regulation service by letting $P_{L}$ go beyond the modified value presented above, this choice may lead to situations when the system would fail to absorb incoming signals in some cases as a result of the limitations in individual power lines. This means that in such situations, in addition to the ordinary sources of error studied in this work (namely the error that occurs when the storage units become fully charged before reaching end of the regulation service period, and the error that occurs when there does not remain enough time for the system to become fully charged by the end of the charging period) we need 
to account for an extra source of error due to the limitations in the individual power lines.

Second, we can generalize the above system to a bidirectional setting. In that case, constraint $m-r \geq 0$ in 5 should be replaced by $m-r \geq-P_{L}$.

Third, we can extend the above system to the setting where the base regulation signal $v(t)$ can have a nonzero (either negative or positive) mean. This would represent situations where the peak power of the regulation up and down signals are identical, but the energy contents are not necessarily the same.

In this work, we used a simple dynamic setting to illustrate the advantage of using dynamic optimization to improve the value of the regulation service provided by the system. However, as discussed in Section 6.2.1, this setting is not optimal. It would be interesting to use techniques from dynamic programming to obtain optimal strategies for dynamic optimization in both stochastic and deterministic settings.

\section{Acknowledgment}

We would like to thank the anonymous reviewer for his very helpful comments.

\section{References}

[1] C. Quinn, D. Zimmerle, and T.H. Bradley, J. Power Sources 195 (2010) 1500-1509. 
[2] S. E. Letendre and W. Kempton, Public Utilities Fortnightly 140 (2002) $16-26$.

[3] W. Kempton and J. Tomic, J. Power Sources 144 (2005) 268-279.

[4] W. Kempton and J. Tomic, J. Power Sources 144 (2005) 280-294.

[5] J. Tomic and W. Kempton, J. Power Sources 168 (2007) 459-468.

[6] Guille C., Gross G., Energy Policy 37 (2009) 4379-4390.

[7] A. Brooks, Vehicle-to-grid demonstration project: grid regulation ancillary service with a battery electric vehicle, California Air Resources Board, 2002.

[8] W. Kempton, et al., A Test of Vehicle-to-grid (V2G) for Energy Storage and Frequency Regulation in the PJM System [Online]. Available: http://www.magicconsortium.org, 2008.

[9] Enbala Power Networks ${ }^{\mathrm{TM}}$, http://www.enbala.com.

[10] S. B. Peterson, J. F. Whitacre, and J. Apt, J. Power Sources 195 (2010) $2377-2384$.

[11] S. B. Peterson, J. Apt, and J. F. Whitacre, J. Power Sources 195 (2010) $2385-2392$.

[12] Brooks, A.; Lu, E.; Reicher, D.; Spirakis, C.; Weihl, B., IEEE Power and Energy Mag. 8 (2010) 20-29. 
[13] http://www.udel.edu/V2G/Tools.html.

[14] Kamboj, S., Decker, K., Trnka, K., Pearre, N., Kern, C. and Kempton, W., Exploring the formation of Electric Vehicle Coalitions for VehicleTo-Grid Power Regulation, AAMAS workshop on Agent Technologies for Energy Systems, 2010.

[15] Sachin Kamboj and Willett Kempton and Keith S. Decker, Deploying Power Grid-Integrated Electric Vehicles as a Multi-Agent System, AAMAS workshop on Agent Technologies for Energy Systems, 2011.

[16] E. Sortomme, M.A., El-Sharkawi, IEEE Trans. Smart Grid 2 (2011) $131-138$.

[17] E. Sortomme, M.A., El-Sharkawi, IEEE Trans. Smart Grid 3 (2012) $70-79$.

[18] E. Sortomme, M.A., El-Sharkawi, IEEE Trans. Smart Grid 3 (2012) $351-359$.

[19] S. Han; S. Han; K. Sezaki, IEEE Trans. on Smart Grid 1 (2010) 65-72.

[20] S. Han; S. Han; K. Sezaki, Stochastic analysis on the energy constraint of V2G frequency regulation, IEEE Vehicle Power and Propulsion Conference (VPPC), 2010.

[21] S. Jang, S. Han; S. Han, K. Sezaki, Optimal Decision on Contract Size for V2G Aggregator regarding Frequency Regulation, 12th International 
Conference on Optimization of Electrical and Electronic Equipment, OPTIM 2010.

[22] Kashyap, A.; Callaway, D., Controlling distributed energy constrained resources for power system ancillary services, IEEE 11th International Conference on Probabilistic Methods Applied to Power Systems (PMAPS), 2010.

[23] N. Rotering, M. Ilic, IEEE Trans. Power Syst. 26 (2011) 1021-1029.

\section{APPENDICES}

Appendix A

In this appendix, we obtain the mean and the standard deviation of the system total residual capacity $S(t)$. The value of $S(t)$ at time $t=l \Delta t$ is given by:

$$
S(t)=S_{0}+\sum_{j=1}^{l} x_{j} \Delta t, \quad 0 \leq t \leq T
$$

Using the linearity of expectation, we can obtain the mean of $S(t)$ as:

$$
\mathbb{E}[S(t)]=S_{0}+\sum_{j=1}^{l} \mathbb{E}\left[x_{j}\right] \Delta t=S_{0}+m l \Delta t=S_{0}+m t, \quad 0 \leq t \leq T
$$


To obtain the variance of $S(t)$, we note that $x_{j}=m+r v_{j}, 1 \leq j \leq k$. Here, $k$ is the total number of regulation signals sent to the fleet. With these notations, we have:

$$
\begin{aligned}
S(t) & =S_{0}+\sum_{j=1}^{l} x_{j} \Delta t \\
& =S_{0}+\sum_{j=1}^{l}\left(m+r v_{j}\right) \Delta t \\
& =S_{0}+m l \Delta t+r \Delta t \sum_{j=1}^{l} v_{j}
\end{aligned}
$$

As a result,

$$
\sigma_{S}^{2}(t)=\operatorname{Var}[S(t)]=(r \Delta t)^{2} \operatorname{Var}\left[\sum_{j=1}^{l} v_{j}\right]
$$

To obtain $\operatorname{Var}\left[\sum_{j=1}^{l} v_{j}\right]$ we note that $\mathbb{E}\left[\sum_{j=1}^{l} v_{j}\right]=\sum_{j=1}^{l} \mathbb{E}\left[v_{j}\right]=0$. Let $R_{v}(i)=\mathbb{E}\left[v_{j} v_{j-i}\right]$ be the autocorrelation function of the zero-mean signal $v$. We have: 


$$
\begin{aligned}
\operatorname{Var}\left[\sum_{j=1}^{l} v_{j}\right] & =\mathbb{E}\left[\left(\sum_{j=1}^{l} v_{j}-\mathbb{E}\left[\sum_{j=1}^{l} v_{j}\right]\right)^{2}\right] \\
& =\mathbb{E}\left[\left(\sum_{j=1}^{l} v_{j}\right)^{2}\right] \\
& =\mathbb{E}\left[\sum_{j=1}^{l} v_{j}^{2}\right]+2 \mathbb{E}\left[\sum_{1 \leq j^{\prime}<j \leq n}^{l} v_{j^{\prime}} v_{j}\right] \\
& =l R_{v}(0)+2 \sum_{1 \leq j^{\prime}<j \leq n}^{l} R_{v}\left(j^{\prime}-j\right) \\
& =l R_{v}(0)+2 \sum_{i=1}^{l-1}(l-i) R_{v}(i) \\
& =l\left[R_{v}(0)+2 \sum_{i=1}^{l-1} R_{v}(i)\right]-2 \sum_{i=1}^{l-1} i R_{v}(i)
\end{aligned}
$$

Plugging the value of $\operatorname{Var}\left[\sum_{j=1}^{l} v_{j}\right]$ from Eq. 32 into 31, we obtain:

$$
\begin{aligned}
\sigma_{S}^{2}(t) & =(r \Delta t)^{2} \operatorname{Var}\left[\sum_{j=1}^{l} v_{j}\right] \\
& =r^{2}(\Delta t)^{2}\left[l\left(R_{v}(0)+2 \sum_{i=1}^{l-1} R_{v}(i)\right)-2 \sum_{i=1}^{l-1} i R_{v}(i)\right] \\
& =r^{2}\left\{(l \Delta t) \Delta t\left[\left(R_{v}(0)+2 \sum_{i=1}^{l-1} R_{v}(i)\right)\right]-2(\Delta t)^{2} \sum_{i=1}^{l-1} i R_{v}(i)\right\} \\
& =r^{2}\left\{t \Delta t\left[\left(R_{v}(0)+2 \sum_{i=1}^{l-1} R_{v}(i)\right)\right]-2(\Delta t)^{2} \sum_{k=1}^{l-1} i R(i)\right\} \\
& =r^{2} \sigma_{0}^{2}(t)
\end{aligned}
$$


where $\sigma_{0}^{2}(t)$ is given by Eq. 8 ,

Appendix $B$

In this appendix, we prove Theorem 2. Let $\left(m, r, T_{0}\right)$ be the optimal solution to 10 (we have dropped the superscript opt for simplicity). To prove Theorem 2, we show that the values of $T_{1}$ and $T_{2}$ corresponding to $(m, r)$ are equal to each other if $g(t)=\frac{\sigma_{0}(t)}{t}$ is a decreasing function of $t$. This is equivalent to saying that both inequalities 11 and 12 hold at their equality under the above condition.

We use proof by contradiction. We assume the optimal solutions of of $m$ and $r$ lead to either $T_{1}<T_{2}$ or $T_{1}>T_{2}$, and show that both these assumptions lead to contradiction.

First, consider the case where $T_{1}<T_{2}$. In this case, we have $T_{0}=$ $\min \left(T_{1}, T_{2}\right)=T_{1}$. In this case, it can be shown that for optimal values of $m$ and $r$, we must have $m-r=0$. Otherwise, if $m-r>0$, we can fix the value of $r$ and replace $m$ by $m^{*}<m$ such that $m^{*}-r$ still remains greater than zero. Now let $T_{1}^{*}$ and $T_{2}^{*}$ denote new crossing points associated with $m^{*}$ and $r$. It is not hard to see that $T_{1}^{*}$ will be larger than $T_{1}$. To see this, we note that the value of $f_{m^{*}, r}^{\mathrm{up}}(t)$ is less than $f_{m, r}^{\mathrm{up}}(t)$ for all $t$ : 


$$
\begin{aligned}
f_{m^{*}, r}^{\mathrm{up}}(t)-f_{m, r}^{\mathrm{up}}(t) & =S_{0}+m^{*} t+\alpha r \sigma_{0}(t)-\left(S_{0}+m t+\alpha r \sigma_{0}(t)\right. \\
& =t\left(m^{*}-m\right) \\
& <0
\end{aligned}
$$

The above inequality holds because $m^{*}<m$ and the time parameter $t$ is always positive. Since $f_{m^{*}, r}^{\mathrm{up}}(t)<f_{m, r}^{\mathrm{up}}(t)$ for all $t$, the crossing point $f_{m^{*}, r}^{\mathrm{up}}(t)$ with the top horizontal line, i.e., $T_{1}^{*}$, always lies at the right of the crossing pint $f_{m, r}^{\text {up }}(t)$ with the same line, i.e., $T_{1}$. Thus, the value of $T_{1}^{*}$ would be greater than $T_{1}$. Because of the continuous nature of the modification in $m$, we can choose $m^{*}$ such that $T_{1}^{*}$ remains less than $T_{2}^{*}$. Under this assumption, the value of $T_{0}^{*}$ defined as $\min \left(T_{1}^{*}, T_{2}^{*}\right)$ will be equal to $T_{1}^{*}$.

Now it can be seen that in the new setting the value of $r$ has not changed and $T_{1}^{*}$ is greater than $T_{1}$. As a result, we have $r T_{1}^{*}>r T_{1}$. Since $T_{0}^{*}=T_{1}^{*}$ and $T_{0}=T_{1}$, this implies $r T_{0}^{*}>r T_{0}$. This is in contradiction with the assumption that the original values of $m$ and $r$ were the optimal ones.

Now let $m-r=0$ or equivalently $r=m$, so the optimal solution can no longer be modified by the operations like the one mentioned above. It can be shown that even in this case the solution can be improved by decreasing $m$, although decreasing $m$ in this case will be at the price of decreasing $r$. To show this, again let $m^{*}<m$ be a new mean value in the system. We define $r^{*}=m^{*}$ to be the new deviation parameter associated with $m^{*}$. As 
before, let $T_{1}^{*}$ and $T_{2}^{*}$ be the crossing points in the new setting. We show that $T_{1}^{*}>T_{1}$. To prove this, we again show that $f_{m^{*}, r^{*}}^{\text {up }}(t)<f_{m, r}^{\text {up }}(t)$ for all $t$ :

$$
\begin{aligned}
f_{m^{*}, r^{*}}^{\text {up }}(t)-f_{m, r}^{\text {up }}(t) & =S_{0}+m^{*} t+\alpha r^{*} \sigma_{0}(t)-\left(S_{0}+m t+\alpha r \sigma_{0}(t)\right) \\
& =S_{0}+m^{*} t+\alpha m^{*} \sigma_{0}(t)-\left(S_{0}+m t+\alpha m \sigma_{0}(t)\right) \\
& =\left(t+\alpha \sigma_{0}(t)\right)\left(m^{*}-m\right) \\
& <0
\end{aligned}
$$

The above inequality comes from the fact that $m^{*}<m$ and the time parameter $t$ and baseline standard deviation $\sigma_{0}(t)$ are positive. It can be seen that since $f_{m^{*}, r^{*}}^{\text {up }}(t)<f_{m, r}^{\text {up }}(t)$, the intersection between $f_{m^{*}, r^{*}}^{\text {up }}(t)$ and the horizontal line at the top of the rectangle lies at the right of the intersection between $f_{m^{*}, r^{*}}^{\text {up }}(t)$ and the same line. That is, $T_{1}^{*}$ is greater than $T_{1}$. Again, because of the continuous nature of the above modification in the mean value, we can choose $m^{*}$ such that $T_{1}^{*}$ remains less than $T_{2}^{*}$, and thus the value of $T_{0}^{*}$, defined as $\min \left(T_{1}^{*}, T_{2}^{*}\right)$, will be equal to $T_{1}^{*}$.

Now we show that $r^{*} T_{1}^{*}>r T_{1}$. To show this, we note that the values of $f_{m^{*}, r^{*}}^{\text {up }}\left(T_{1}^{*}\right)$ and $f_{m, r}^{\text {up }}\left(T_{1}\right)$ are both equal to $C$ and thus equal to each other:

$$
S_{0}+m^{*} T_{1}^{*}+\alpha r^{*} \sigma_{0}\left(T_{1}^{*}\right)=S_{0}+m T_{1}+\alpha r \sigma_{0}\left(T_{1}\right)
$$

Using $m^{*}=r^{*}$ and $m=r$, and removing $S_{0}$ from both sides, we can 
simplify the above equation as follows:

$$
r^{*} T_{1}^{*}+\alpha r^{*} \sigma_{0}\left(T_{1}^{*}\right)=r T+\alpha r \sigma_{0}\left(T_{1}\right)
$$

Now we rewrite the above equality as:

$$
r^{*} T_{1}^{*}\left(1+\alpha \frac{\sigma_{0}\left(T_{1}^{*}\right)}{T_{1}^{*}}\right)=r T_{1}\left(1+\alpha \frac{\sigma_{0}\left(T_{1}\right)}{T_{1}}\right)
$$

or,

$$
\frac{r^{*} T_{1}^{*}}{r T_{1}}=\frac{1+\alpha \frac{\sigma_{0}\left(T_{1}\right)}{T_{1}}}{1+\alpha \frac{\sigma_{0}\left(T_{1}^{*}\right)}{T_{1}^{*}}}
$$

Since $\frac{\sigma_{0}(t)}{t}$ is a assumed to be a decreasing function of $t$ and $T_{1}^{*}>T_{1}$, the right-hand side of the above equality is greater than one and so is the left-hand side. As a result, we have $r^{*} T_{1}^{*}>r T$. The facts that $T_{0}^{*}=T_{1}^{*}$ and $T_{0}=T_{1}$ imply $r^{*} T_{0}^{*}>r T_{0}$. This is in contradiction with the assumption that the values of $m$ and $r$ are the optimal solutions to the problem.

Next, we consider the case where $T_{1}>T_{2}$ for the optimal solutions $m$ and $r$, and again show this is in contradiction with the optimality of the solution. In this case, $T_{0}=\min \left(T_{1}, T_{2}\right)=T_{2}$. It can be shown that under the above assumption, we must have $m+r=P_{L}$ for the optimal values of $m$ and $r$. Otherwise, similar to the procedure mentioned in the previous case, we can fix the value of $r$ and increase the value of $m$, such that the objective function is increased. 
Next we assume $m+r=P_{L}$. We show that under this assumption, if we increase the mean value $m$, which will be at the expense of reducing $r$, the overall objective function will be increased. To show this, let $m^{*}>m$ be the new mean value and $r^{*}=P_{L}-m^{*}$ be the associated deviation parameter. We show $f_{m^{*}, r^{*}}^{\text {low }}(t)>f_{m, r}^{\text {low }}(t)$ for all $t$. To see this, we note:

$$
\begin{aligned}
f_{m^{*}, r^{*}}^{\text {low }}(t)-f_{m, r}^{\text {low }}(t)= & S_{0}+m^{*} t-\alpha r^{*} \sigma_{0}(t)-\left(S_{0}+m t-\alpha r \sigma_{0}(t)\right) \\
= & S_{0}+m^{*} t-\alpha\left(P_{L}-m\right) \sigma_{0}(t)- \\
& \left(S_{0}+m t-\alpha\left(P_{L}-m\right) \sigma_{0}(t)\right) \\
= & t\left(m^{*}-m\right)+\alpha \sigma_{0}(t)\left(m^{*}-m\right) \\
= & \left(t+\alpha \sigma_{0}(t)\right)\left(m^{*}-m\right) \\
> & 0
\end{aligned}
$$

Since $f_{m^{*}, r^{*}}^{\text {low }}(t)>f_{m, r}^{\text {low }}(t)$, the intersection between $f_{m^{*}, r^{*}}^{\text {low }}(t)$ and the line with slope $P_{L}$ at the right-hand side of the geometric representation lies at the right of the intersection between $f_{m, r}^{\text {low }}(t)$ and the same line. Thus, we have $T_{2}^{*}>T_{2}$. Again note that, because of the continuous nature of the above change in $m$, the value of $m^{*}$ can be chosen such that $T_{2}^{*}$ remains less than $T_{1}^{*}$. Under this assumption, the value of $T_{0}^{*}$ defined as $\min \left(T_{1}^{*}, T_{2}^{*}\right)$ will be equal to $T_{2}^{*}$.

Now we show that $r^{*} T_{2}^{*}>r T_{2}$. To show this, we note that the values of $\frac{C-f_{m^{*}, r^{*}}^{\text {low }}\left(T_{2}^{*}\right)}{P_{L}}-\left(T-T_{2}^{*}\right)$ and $\frac{C-f_{m, r}^{\text {low }}\left(T_{2}\right)}{P_{L}}-\left(T-T_{2}\right)$ are both equal to zero, and 
thus equal to each other. So we have:

$$
\begin{aligned}
\frac{C-\left(S_{0}+m^{*} T_{2}^{*}-\alpha r^{*} \sigma_{0}\left(T_{2}^{*}\right)\right)}{P_{L}}-\left(T-T_{2}^{*}\right)= \\
\quad \frac{C-\left(S_{0}+m T_{2}-\alpha r \sigma_{0}\left(T_{2}\right)\right)}{P_{L}}-\left(T-T_{2}\right)
\end{aligned}
$$

Using $m^{*}=P_{L}-r^{*}$ and $m=P_{L}-r$, and simplifying the above equality, we obtain:

$$
r^{*} T_{2}^{*}+\alpha r^{*} \sigma_{0}\left(T_{2}^{*}\right)=r T_{2}+\alpha r \sigma_{0}\left(T_{2}\right)
$$

The above equality can be written as follows:

$$
r^{*} T_{2}^{*}\left(1+\alpha \frac{\sigma_{0}\left(T_{2}^{*}\right)}{T_{2}^{*}}\right)=r T_{2}\left(1+\alpha \frac{\sigma_{0}\left(T_{2}\right)}{T_{2}}\right)
$$

or,

$$
\frac{r^{*} T_{2}^{*}}{r T_{2}}=\frac{\left(1+\alpha \frac{\sigma_{0}\left(T_{2}\right)}{T_{2}}\right)}{\left(1+\alpha \frac{\sigma_{0}\left(T_{2}^{*}\right)}{T_{2}^{*}}\right)}
$$

Since $\frac{\sigma_{0}(t)}{t}$ is a assumed to be a decreasing function of $t$ and $T_{2}^{*}>T_{2}$, the right-hand side of the above equality is greater than one and so is the left-hand side. As a result, we have $r^{*} T_{2}^{*}>r T_{2}$. Since $T_{0}^{*}=T_{2}^{*}$ and $T_{0}=T_{2}$, this implies that $r^{*} T_{0}^{*}>r T_{0}$. This result is again in contradiction with 
the assumption that the values of $m$ and $r$ are the optimal solutions to the problem.

\section{Appendix $C$}

In this appendix, we prove Theorem 3. In proving this theorem, we assume regulation signals and the system residual capacity can be approximated by continuous signals. This is a reasonable assumption as the time span between regulation signals, $\Delta t$, is much smaller than other time scales in the problem. With this approximation, Eq. 4 becomes:

$$
S(t)=S_{0}+\Delta t \sum_{j=1}^{\lfloor t / \Delta t\rfloor} x_{j}=S_{0}+\int_{0}^{t} x(\tau) d \tau=S_{0}+m t+r \int_{0}^{t} v(\tau) d \tau
$$

In this continuous form, variance of $S(t)$ is given by $\sigma_{S}^{2}(t)=r^{2} \sigma_{0}^{2}(t)$, where:

$$
\sigma_{0}^{2}(t)=2 t \int_{0}^{t} R_{v}(\tau) d \tau-2 \int_{0}^{t} \tau R_{v}(\tau) d \tau
$$

To show the above equation, one can expand variance of $S(t)$ in 35 via a double integral and use change of variables to obtain the final result. An alternative way to obtain Eq. 36 is to show that Eq. 36 is equivalent to Eq. 8 when $\Delta t \ll t$.

Now we show that if $R_{v}(\tau)>0$ for all $\tau$ then function $g(t)=\frac{\sigma_{0}(t)}{t}$ will be a decreasing function of $t$. Since $g(t)$ is always positive we can equivalently show that $f(t)=g^{2}(t)=\frac{\sigma_{0}^{2}(t)}{t^{2}}$ is a decreasing function of $t$. To show that 
$f(t)$ is a decreasing function of $t$, we show that its derivative is negative for all $t>0$. Let $h(t)=\sigma_{0}^{2}(t)$. Then, $f(t)=\frac{h(t)}{t^{2}}$, and the derivative of $f(t)$ will be:

$$
f^{\prime}(t)=\frac{h^{\prime}(t) t^{2}-h(t)(2 t)}{t^{4}}=\frac{t h^{\prime}(t)-2 h(t)}{t^{3}}
$$

The denominator in Eq. 37 is always positive when $t>0$. Thus, to show $f^{\prime}(t)<0$ it suffices to show that the numerator is negative for $t>0$. By taking derivative of Eq. [36, we obtain $h^{\prime}(t)=2 \int_{0}^{t} R(\tau) d \tau$. Substituting the values of $h(t)$ and $h^{\prime}(t)$ in the nominator of Eq. 37, we obtain:

$$
\begin{aligned}
t h^{\prime}(t)-2 h(t) & =2 t \int_{0}^{t} R(\tau) d \tau-\left(4 t \int_{0}^{t} R(\tau) d \tau-2 \int_{0}^{t} \tau R(\tau) d \tau\right) \\
& =2\left(\int_{0}^{t} \tau R(\tau) d \tau-\int_{0}^{t} t R(\tau) d \tau\right)
\end{aligned}
$$

Note that $\tau \leq t$ for all $\tau \in(0, t)$, and thus for positively-valued functions $R(\tau), \tau R(\tau) \leq t R(\tau), \tau \in(0, t)$. This implies that the right-hand side of Eq. 38 is negative, as desired.

Appendix D

In this appendix, we prove Theorem 3 . We first note that the optimization problem 5 in the deterministic case becomes: 


$$
\begin{array}{cc}
\max _{m, r, T_{0}} & r T_{0} \\
\text { s.t. } & m, r, T_{0} \geq 0, T_{0} \leq T \\
m-r \geq 0 \\
m+r \leq P_{L} \\
S_{0}+(m+r) T_{0} \leq C \\
\frac{C-\left[S_{0}+(m-r) T_{0}\right]}{P_{L}} \leq T-T_{0}
\end{array}
$$

We consider two cases:

Case 1. $Q \leq 1$ : We first show that in this case $T_{2} \geq T_{1}$ in any optimal solution of the problem. To show this we use proof by contradiction. We first note that if $T_{2}<T_{1}$ in an optimal solution, then $m+r$ must equal to $P_{L}$. Otherwise, one can fix $r$ and increase $T_{0}$ (here equal to $T_{2}$ ) by slightly increasing the value of $m$ such that $T_{2}$ increases and still remains less than $T_{1}$. This contradicts the assumption that the solution is optimal. Now we note that $Q \leq 1$ and $m+r=P_{L}$ imply $T_{1} \leq T_{2}$. To show this we note that if $m+r=P_{L}$ then $T_{1}$ will be given by the intersection between the line with slope $P_{L}$ at the left-hand side of Fig. 1 and the horizontal line at the top of the diagram. Since $Q \leq 1$ this intersection is always at the left-hand side of the line with slope $P_{L}$ at the right-hand side of the figure, and thus $T_{1} \leq T_{2}$. This contradicts the original assumption that $T_{2}<T_{1}$.

Note that $T_{2} \geq T_{1}$ implies $T_{0}=T_{1}$, and that the inequality in 42 holds 
at equality.

Next, we show that if $T_{2} \geq T_{1}$ then $m-r=0$. This is simple to verify. Because if $m-r>0$, one can increase the value of $T_{0}$ (which is equal to $T_{1}$ in this case) by fixing the value of $r$ and slightly decreasing the value of $m$ such that $T_{1}$ increases and still remains less than $T_{2}$. Again, this contradicts optimality of the solution. Given $m-r=0$, we can replace $r$ by $m$ in 41 and arrive at:

$$
m \leq \frac{P_{L}}{2}
$$

This inequality provides the upper limit of $m$ provided in the first part of Theorem 4 .

To obtain the lower limit, we note that inequality 42 holds at equality when $Q \leq 1$, and thus:

$$
T_{0}=\frac{C-S_{0}}{2 m}
$$

Plugging the value of $T_{0}$ in 43 , we obtain:

$$
m \geq \frac{P_{C}}{2} \frac{1}{1-\frac{P_{C}}{P_{L}}}
$$

Finally, from Eq. 45 and the fact that $r=m$, it can be seen that at any optimal solution, the value of objective function is equal to $\frac{C-S_{0}}{2}$.

Case 2. $Q>1$ : Using procedures similar to the ones presented above, it can be shown that when $Q>1, T_{2} \leq T_{1}$ and $m+r=P_{L}$. The proofs are 
similar and are not presented them for the sake of brevity. Note that $T_{2} \leq T_{1}$ implies $T_{0}=T_{2}$ and thus the inequality in 43 holds at equality.

Setting $r=P_{L}-m$ in 40 implies:

$$
m \geq \frac{P_{L}}{2}
$$

Also, we can set $r=P_{L}-m$ in 43 to obtain:

$$
T_{0}=\frac{T P_{L}-C+S_{0}}{2\left(P_{L}-m\right)}
$$

Plugging the value of $T_{0}$ in 42 , we arrive at:

$$
m \leq \frac{P_{L}}{2} \frac{3 \frac{P_{C}}{P_{L}}-1}{\frac{P_{C}}{P_{L}}}
$$

Inequalities 47 and 49 provide, respectively, the lower and upper limits of $m$ in the second part of Theorem 4 .

From Eq. 48 it can be seen that the value of objective function at all optimal solutions is equal to $\frac{P_{L} T-C+S_{0}}{2}$. 


\section{List of Figures}

Fig. 1 Geometric representation of a typical single-shot charging process.

Fig. 2 Three different regimes for solving the optimization problem (a)

Phase (i) $Q<1$. (b) Phase (ii). $Q>1$ (c) Phase (iii) $Q=1$.

Fig. 3 Optimal value of the regulation service as a function of line peak power $P_{L}$.

Fig. 4 Optimal value and duration of the regulation service as a function of system residual capacity. (a) Optimal value of the regulation service. (b) Optimal duration of the regulation service.

Fig. 5 Optimal value of the regulation service as a function of the correlation time of the regulation signals.

Fig. 6 Optimal value of the regulation service as a function of the variance of the base regulation signal $\sigma_{v}$.

Fig. 7 Geometric representation of a typical dynamic charging process.

Fig. 8 Regulation service as a function of the number of updates.

Fig. 9 QQ plot of the PJM regulation traces versus standard normal.

Fig. 10 Autocorrelation functions of the regulation traces obtained from PJM.

The data is provided for four different days.

Fig. 11 Testing the single-shot charging scheme using PJM regulation traces.

Fig. 12 Testing the dynamic charging scheme using the PJM regulation 
traces. 
Figure 1: Geometric representation of a typical single-shot charging process.

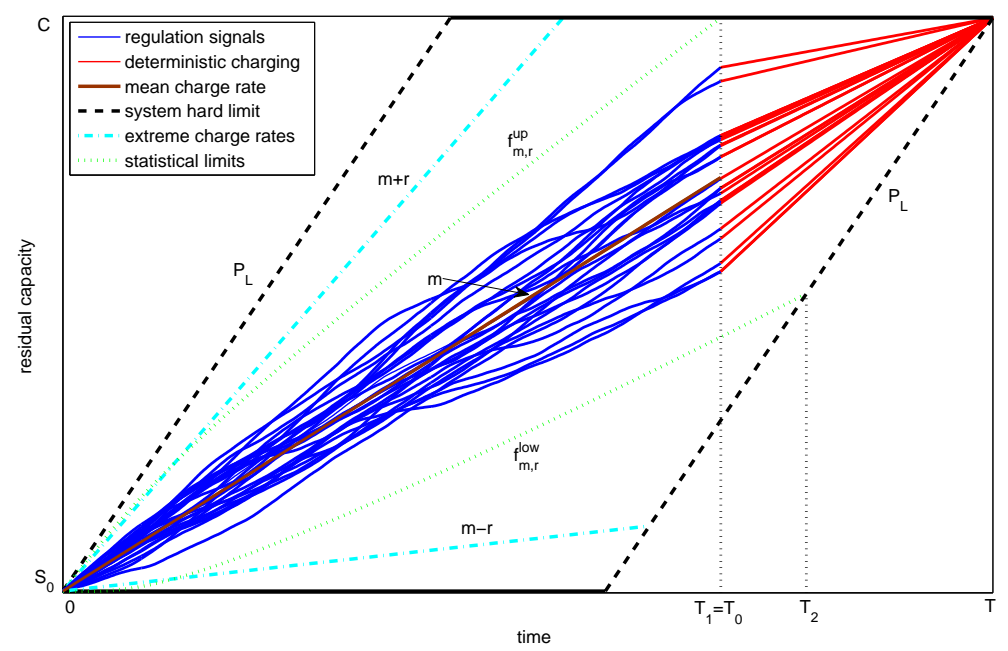


Figure 2: Three different regimes for solving the optimization problem 14.

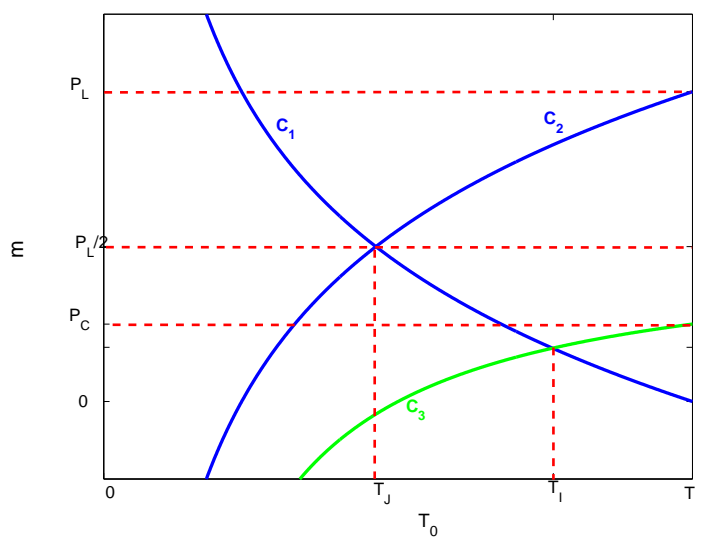

(a) Phase (i) $Q<1$

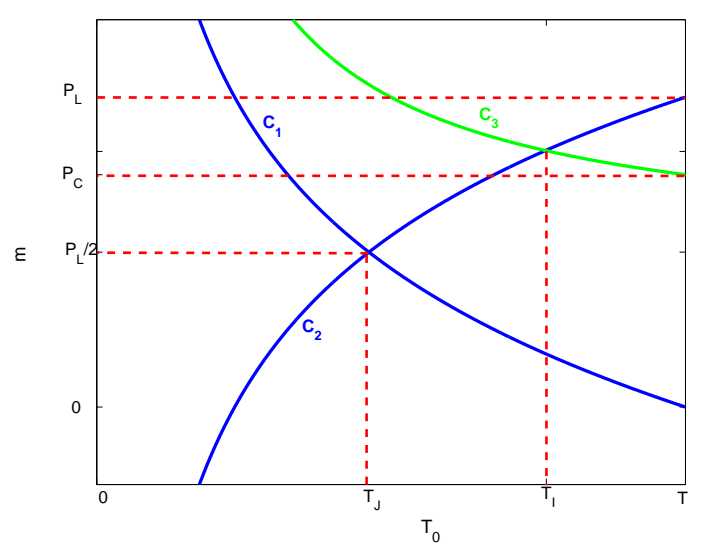

(b) Phase (ii) $Q>1$

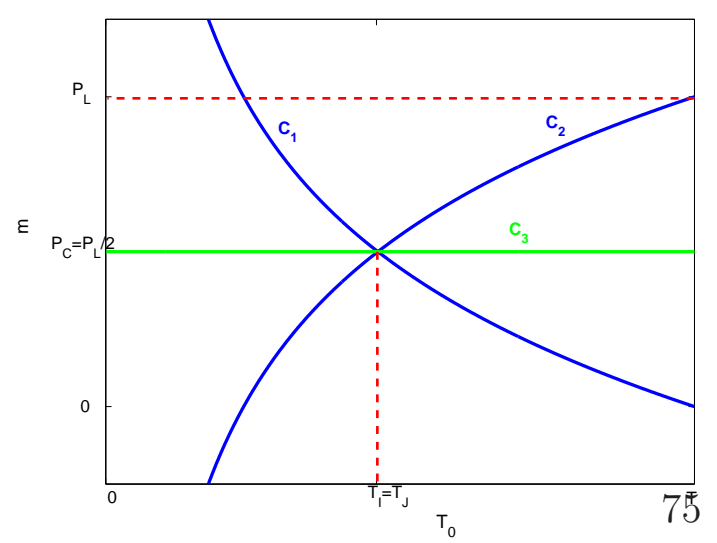

(c) Phase (iii) $Q=1$ 
Figure 3: Optimal value of the regulation service as a function of line peak power $P_{L}$.

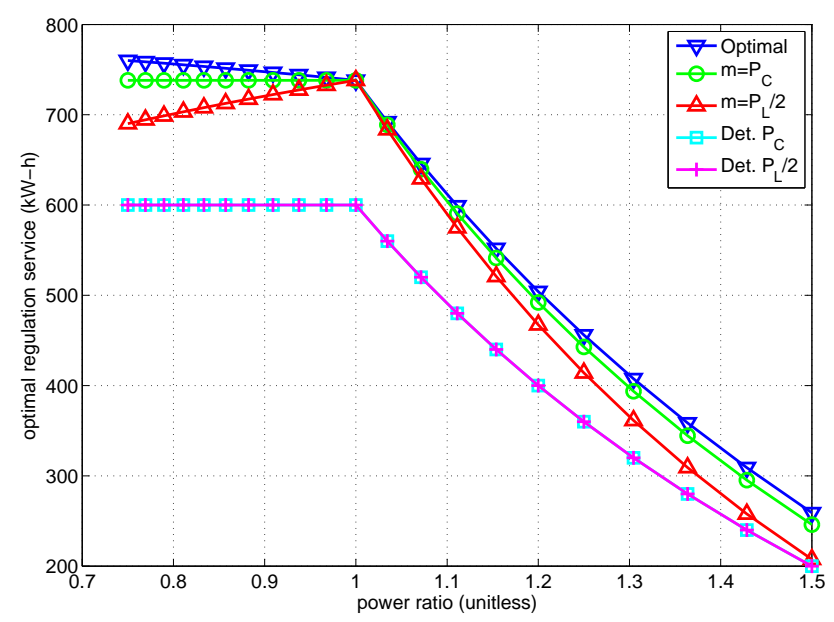

Figure 4: Optimal value and duration of the regulation service as a function of system residual capacity.
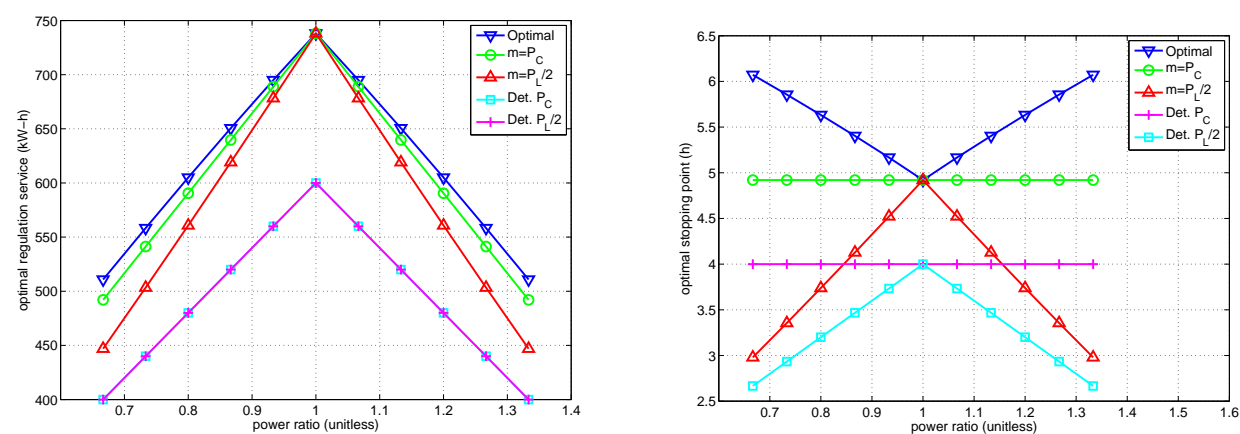

(a) Optimal value of the regulation service.(b) Optimal duration of the regulation service. 
Figure 5: Optimal value of the regulation service as a function of the correlation time of the regulation signals.

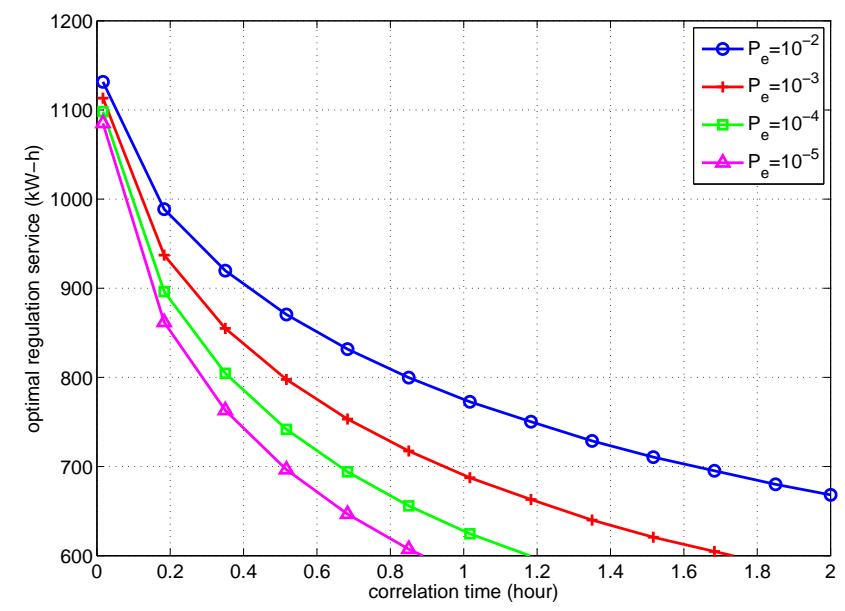

Figure 6: Optimal value of the regulation service as a function of the variance of the base regulation signal $\sigma_{v}$.

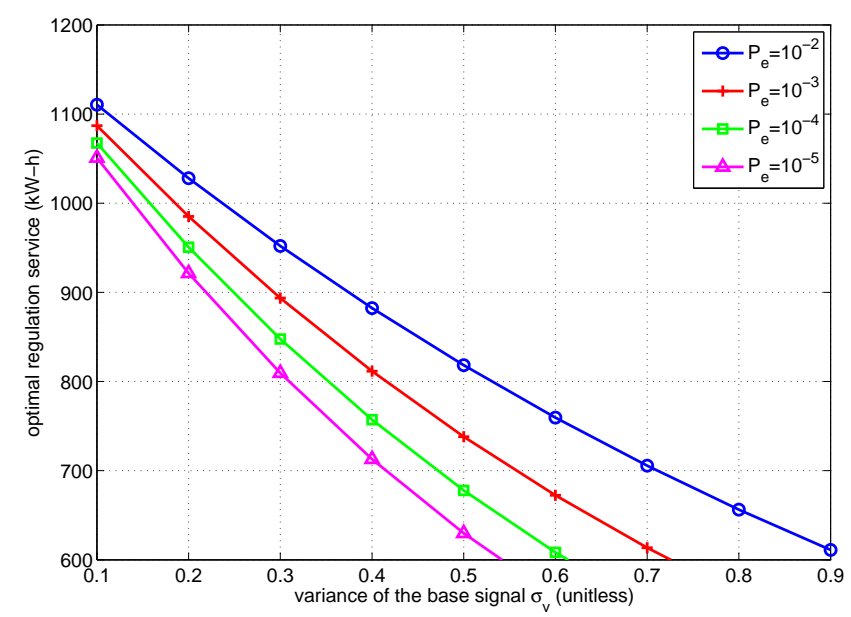


Figure 7: Geometric representation of a typical dynamic charging process.

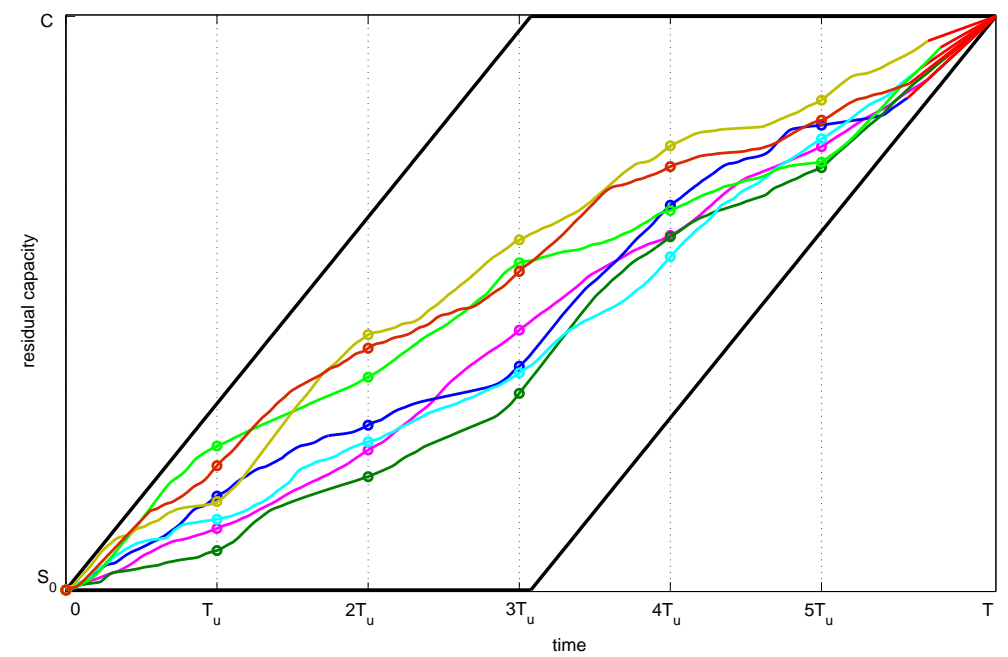

Figure 8: Regulation service as a function of the number of updates.

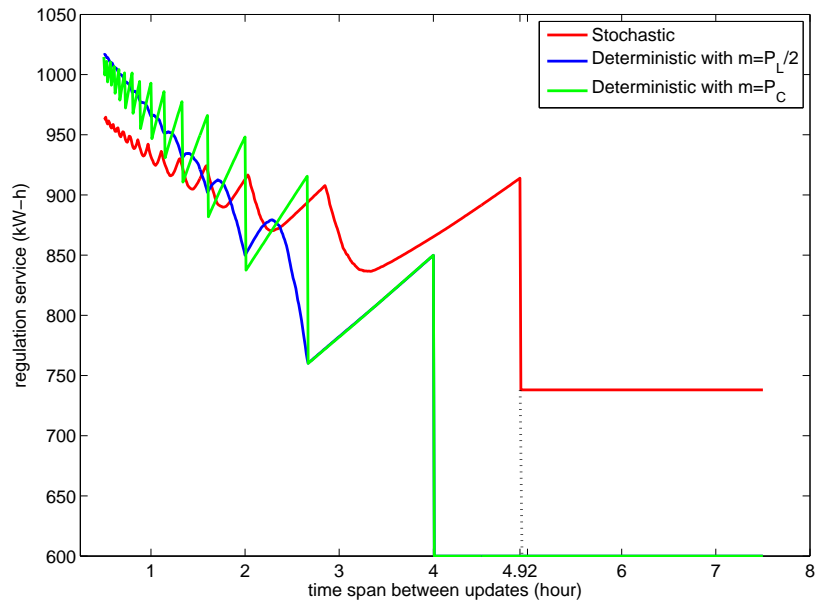


Figure 9: QQ plot of the PJM regulation traces versus standard normal.

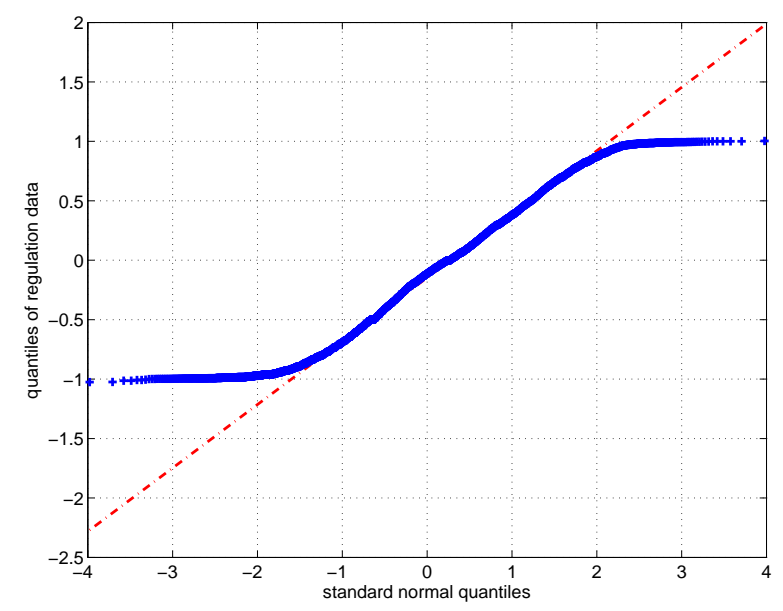

Figure 10: Autocorrelation functions of the regulation traces obtained from PJM. The data is provided for four different days.
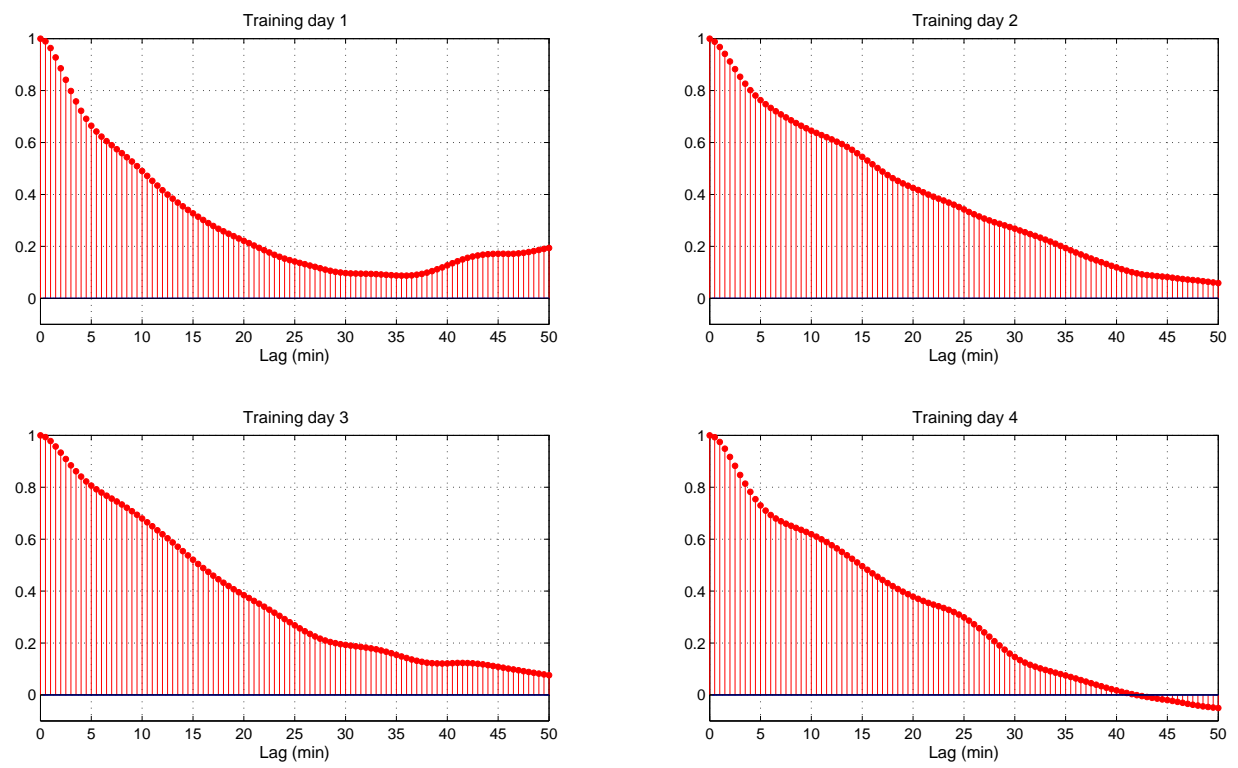
Figure 11: Testing the single-shot charging scheme using PJM regulation traces.

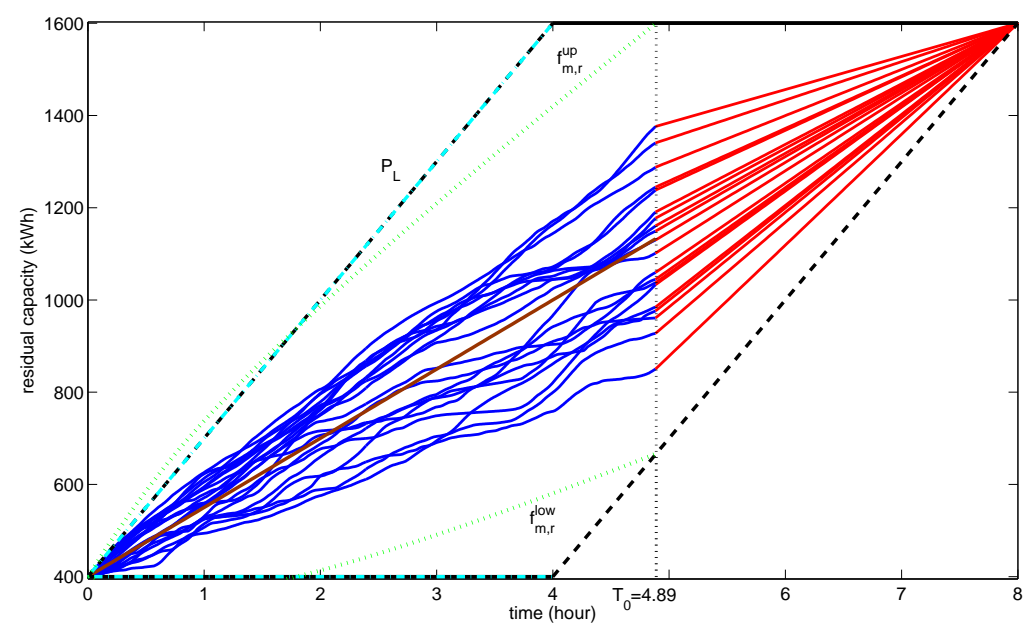

Figure 12: Testing the dynamic charging scheme using the PJM regulation traces.

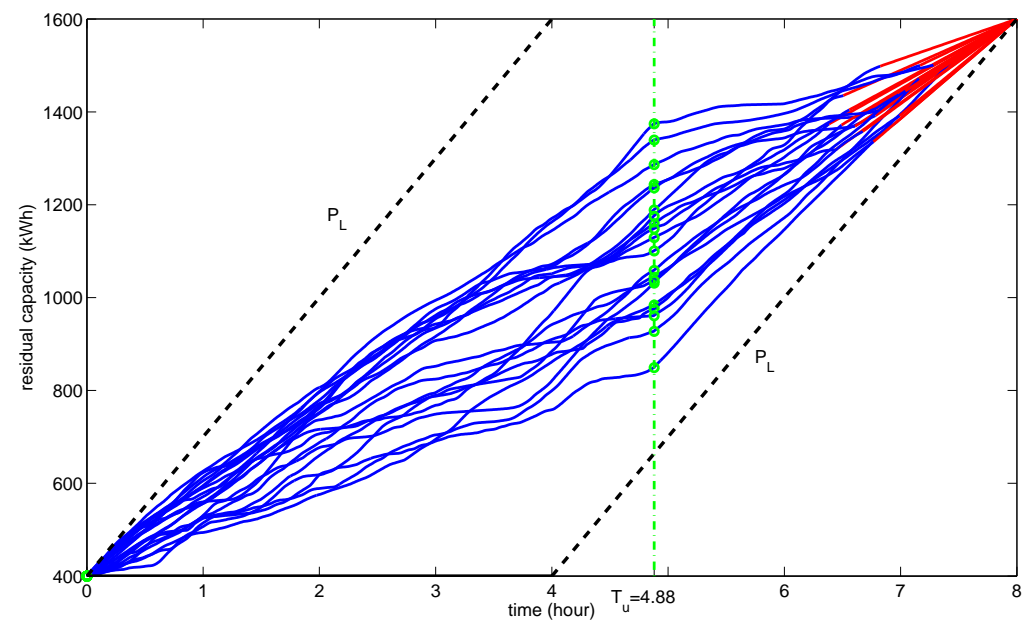

